\title{
Symmetric and quasi-symmetric functions associated to polymatroids
}

\author{
Harm Derksen
}

Received: 21 April 2008 / Accepted: 26 August 2008 / Published online: 9 October 2008

(C) Springer Science+Business Media, LLC 2008

\begin{abstract}
To every subspace arrangement $\mathbf{X}$ we will associate symmetric functions $\mathcal{P}[\mathbf{X}]$ and $\mathcal{H}[\mathbf{X}]$. These symmetric functions encode the Hilbert series and the minimal projective resolution of the product ideal associated to the subspace arrangement. They can be defined for discrete polymatroids as well. The invariant $\mathcal{H}[\mathbf{X}]$ specializes to the Tutte polynomial $\mathcal{T}[\mathbf{X}]$. Billera, Jia and Reiner recently introduced a quasisymmetric function $\mathcal{F}[\mathbf{X}]$ (for matroids) which behaves valuatively with respect to matroid base polytope decompositions. We will define a quasi-symmetric function $\mathcal{G}[\mathbf{X}]$ for polymatroids which has this property as well. Moreover, $\mathcal{G}[\mathbf{X}]$ specializes to $\mathcal{P}[\mathbf{X}], \mathcal{H}[\mathbf{X}], \mathcal{T}[\mathbf{X}]$ and $\mathcal{F}[\mathbf{X}]$.
\end{abstract}

Keywords Matroids · Polymatroids · Symmetric function · Quasi-symmetric function · Tutte polynomial - Subspace arrangement · Hyperplane arrangement

\section{Introduction}

\subsection{Combinatorial invariants}

Let $X$ be a set with $d$ elements. Suppose that $V_{x}, x \in X$ are subspaces of an $n$ dimensional vector space. Then $\mathcal{A}=\bigcup_{x \in X} V_{x}$ is called a subspace arrangement. Let $\operatorname{Pow}(X)$ be the set of all subsets of $X$. The rank function rk: $\operatorname{Pow}(X) \rightarrow \mathbb{N}:=$ $\{0,1,2, \ldots\}$ is defined by

$$
\operatorname{rk}(A)=\operatorname{dim} V-\operatorname{dim} \bigcap_{i \in A} V_{i}
$$

The author is partially supported by the NSF, grant DMS 0349019.

H. Derksen $(\bowtie)$

Department of Mathematics, University of Michigan, East Hall, 530 Church Street, Ann Arbor, MI 48109-1043, USA

e-mail: hderksen@umich.edu 
for all subsets $A \subseteq X$.

Surprisingly, many topological invariants of the complement $V \backslash \mathcal{A}$ of subspace arrangements are combinatorial, i.e., they can be expressed in terms of $n:=\operatorname{dim} V$ and the rank function. For example, Zaslavsky (see [40]) proved that number of regions in the complement of a real hyperplane arrangement is equal to

$$
(-1)^{n} \chi(-1)=\sum_{A \subseteq X}(-1)^{\mathrm{rk}(A)+|A|},
$$

where $\chi(q)$ is the characteristic polynomial of the hyperplane arrangement defined by

$$
\chi(q)=\sum_{A \subseteq X} q^{n-\mathrm{rk}(A)}(-1)^{|A|} .
$$

For complex hyperplane arrangements, the cohomology ring $H^{\star}(V \backslash \mathcal{A})$ is isomorphic to the Orlik-Solomon algebra (see [29]), which is defined explicitly in terms of the rank function. For arbitrary real subspace arrangements, the topological Betti numbers of the complement $V \backslash \mathcal{A}$ are expressed in terms of the rank function using the Goresky-MacPherson formula (see [17]).

One may wonder whether various algebraic objects associated to a subspace arrangements are combinatorial invariants. Let $K$ be a base field of characteristic 0 , and denote the coordinate ring of $V$ by $K[V]$. Terao defined the module of derivations $D(\mathcal{A})$ along a hyperplane arrangement $\mathcal{A}$ (see [36]). An arrangement is called free if $D(\mathcal{A})$ is a free $K[V]$-module. Terao has conjectured that "freeness" is a combinatorial property, i.e., whether $D(\mathcal{A})$ is free is determined by its rank function. Terao showed that free arrangements have the property that their characteristic polynomial factors into linear polynomials (see [36]). One should point out that for example the Hilbert series of the module $D(\mathcal{A})$ is not a combinatorial invariant.

In a recent paper, the author found an algebraic object which is a combinatorial invariant for subspace arrangements. Let $J_{x} \subseteq K[V]$ be the vanishing ideal of $V_{x} \subseteq V$ and let $J=\prod_{x \in X} J_{x}$ be the product ideal. The author showed in [12] that the Hilbert series $H(J, t)$ of $J$ is a combinatorial invariant. For hyperplane arrangements the Hilbert series of $J$ is always equal to $t^{d} /(1-t)^{n}$ and is therefore not an interesting invariant. Let $W$ be an arbitrary vector space and denote its dual by $W^{\star}$. We can tensor all the spaces with $W^{\star}$. So let $J_{x}(W) \subseteq K\left[V \otimes W^{\star}\right]$ be the vanishing ideal of the subspace $V_{x} \otimes W^{\star}$ of $V \otimes W^{\star}$ and $J(W)=\prod_{x \in A} J_{x}(W)$. Then the Hilbert series $H(J(W), t)$ is an interesting invariant, even for hyperplane arrangements. Moreover, since we have an action of GL(W) on all the rings and ideals involved, we can define a $\mathrm{GL}(W)$-equivariant Hilbert series which is a more refined invariant for subspace arrangement.

\subsection{Symmetric functions}

The ring of symmetric functions is spanned by the Schur symmetric functions $s_{\lambda}$ where $\lambda$ runs over all partitions. Let $\mathbf{X}=(X, \mathrm{rk})$ where rk is the rank function coming from a subspace arrangement $\bigcup_{x \in X} V_{x} \subseteq V$. In Section 2.3, we will define a 
symmetric function $\mathcal{P}[\mathbf{X}]$ using a recursive formula (see Definition 2.3). We define another symmetric function $\mathcal{H}[\mathbf{X}]=\mathcal{H}[\mathbf{X}](q, t)$ with coefficients in $\mathbb{Z}[q, t]$ by

$$
\mathcal{H}[\mathbf{X}](q, t)=\sum_{A \subseteq X} \mathcal{P}\left[\left.\mathbf{X}\right|_{A}\right] q^{\mathrm{rk}(A)} t^{|A|}
$$

Here $\left.\mathbf{X}\right|_{A}=\left(A,\left.\mathrm{rk}\right|_{A}\right)$ can be viewed as the rank function of the sub-arrangement $\bigcup_{x \in A} V_{x} \subseteq V$. The definitions of $\mathcal{P}[\mathbf{X}]$ and $\mathcal{H}[\mathbf{X}](q, t)$ make sense even if the rank function rk does not come from a subspace arrangement. Therefore, these symmetric functions can also be defined for polymatroids. The symmetric function $\mathcal{H}[\mathbf{X}](q, t)$ essentially encodes Hilbert series of $J$ and the GL( $W)$-equivariant Hilbert series of $J(W)$. Also, the minimal free resolutions of $J$ and $J(W)$ can be expressed in terms of $\mathcal{H}[\mathbf{X}](q, t)$. The symmetric functions behave nicely with respect to direct sums of polymatroids, namely

$$
\begin{aligned}
\mathcal{P}[\mathbf{X} \oplus \mathbf{Y}] & =\mathcal{P}[\mathbf{X}] \cdot \mathcal{P}[\mathbf{Y}] \\
\mathcal{H}[\mathbf{X} \oplus \mathbf{Y}](q, t) & =\mathcal{H}[\mathbf{X}](q, t) \cdot \mathcal{H}[\mathbf{Y}](q, t)
\end{aligned}
$$

(see Proposition 2.6). The Tutte polynomial is defined by

$$
\mathcal{T}[\mathbf{X}](x, y)=\sum_{A \subseteq X}(x-1)^{\operatorname{rk}(X)-\operatorname{rk}(A)}(y-1)^{|A|-\operatorname{rk}(A)} .
$$

The Tutte polynomial was introduced in [37] and generalized to matroids in [4] and [8]. It has the multiplicative property and it behaves well under matroid duality (see (5)). It specializes to the characteristic polynomial, namely

$$
\chi(q)=q^{n-\mathrm{rk}(X)} \mathcal{T}[\mathbf{X}](1-q, 0) .
$$

The coefficients of $\mathcal{T}[\mathbf{X}](x, y)$ as a polynomial in $x$ and $y$ have combinatorial interpretations and are nonnegative. The invariant $\mathcal{H}[\mathbf{X}](q, t)$ specializes to the Tutte polynomial. The functions $\mathcal{P}[\mathbf{X}]$ and $\mathcal{H}[\mathbf{X}](q, t)$ do not seem to behave nicely under matroid duality. If the polymatroid $\mathbf{X}$ is realizable as a subspace arrangement in characteristic 0 , then the coefficients of $\mathcal{P}[\mathbf{X}], \mathcal{H}[\mathbf{X}](q, t)$ and some of their specializations have homological interpretations. Therefore, the coefficients of these functions satisfy certain non-negativity conditions.

Brylawski defined a graph invariant in [5] which he called the polychromate. Sarmiento [31] proved that the polychromate is equivalent to the $U$-polynomial studied by Noble and Welch [28]. The polychromate and the U-polynomial specialize to Stanley's chromatic symmetric polynomial [35]. There are graphs whose graphical matroids are the same, that can be distinguished by the Stanley symmetric function. This means that the Stanley symmetric function, the polychromatic, and the U-polynomial cannot be viewed as invariants of matroids.

Inspired by these graph invariants, Billera, Jia and Reiner defined a quasisymmetric function which $i s$ an invariant for matroids (see [3]). This invariant will be discussed later. 


\subsection{Polarized Schur functions}

Let us denote the Schur functor corresponding to the partition $\lambda$ by $S_{\lambda}$. Suppose our base field $K$ has characteristic $0, Z$ is a finite dimensional $K$-vector space, and $Z_{1}, \ldots, Z_{d} \subseteq Z$ are subspaces. For a partition $\lambda$ with $|\lambda|=d$ we will define a subspace

$$
S_{\lambda}\left(Z_{1}, Z_{2}, \ldots, Z_{d}\right) \subseteq S_{\lambda}(Z)
$$

as the subspace spanned by the all $\pi\left(z_{1} \otimes \cdots \otimes z_{d}\right)$ where $z_{i} \in Z_{i}$ for all $i$ and

$$
\pi: \underbrace{Z \otimes Z \otimes \cdots \otimes Z}_{d} \rightarrow S_{\lambda}(Z)
$$

is a $\mathrm{GL}(Z)$-equivariant linear map.

The space $S_{\lambda}\left(Z_{1}, \ldots, Z_{d}\right)$ has various interesting properties which will be discussed in Section 6. For example

$$
S_{\lambda}(\underbrace{Z, Z, \ldots, Z}_{d})=S_{\lambda}(Z) .
$$

Also, permuting the spaces $Z_{1}, \ldots, Z_{d}$ does not change the subspace $S_{\lambda}\left(Z_{1}, \ldots, Z_{d}\right)$. Let $V=Z^{\star}$ be the dual space, and define $V_{i}=Z_{i}^{\perp}$ to be the subspace of $V$ orthogonal to $Z_{i}$. Consider the subspace arrangement $\mathcal{A}=V_{1} \cup \cdots \cup V_{d} \subseteq V$. Then the dimension of $S_{\lambda}\left(Z_{1}, \ldots, Z_{d}\right)$ can be expressed in terms of $\mathcal{H}[\mathcal{A}](q, t)$. This implies, that the dimension of $S_{\lambda}\left(Z_{1}, \ldots, Z_{d}\right)$ is determined by the numbers

$$
\operatorname{dim} \sum_{i \in A} Z_{i}, \quad A \subseteq\{1,2, \ldots, d\} .
$$

\subsection{Quasi-symmetric functions}

Billera, Jia and Reiner defined a quasi-symmetric function $\mathcal{F}[\mathbf{X}]$ for any matroid $\mathbf{X}$ in [3]. This invariant behaves nicely with respect to direct sums of matroids, matroid duality. There is also a very natural definition of this invariant in terms of the combinatorial Hopf algebras studied in [1] (see Section 7.4). In [3] it was proved that this quasi-symmetric function behaves valuatively with respect to matroid polytope decompositions, so it can be a useful tool for studying such decompositions. The quasi-symmetric $\mathcal{F}[\mathbf{X}]$ does not specialize to $\mathcal{H}[\mathbf{X}](q, t)$ because $\mathcal{F}[\mathbf{X}]$ cannot distinguish between a loop or an isthmus, and $\mathcal{H}[\mathbf{X}](q, t)$ can. We will show that $\mathcal{F}[\mathbf{X}]$ does specialize to $\mathcal{P}[\mathbf{X}]$. To prove this, we introduce another quasi-symmetric function $\mathcal{G}[\mathbf{X}]$ which should be of interest on its own right. First of all, we will choose a convenient basis $\left\{U_{r}\right\}$ of the ring of quasi-symmetric functions where $r$ runs over all finite sequences of nonnegative integers. A complete chain is a sequence

$$
\underline{X}: \varnothing=X_{0} \subset X_{1} \subset \cdots \subset X_{d}=X
$$

such that $X_{i}$ has $i$ elements for all $i$. The rank vector of this chain $\underline{X}$ is defined by

$$
r(\underline{X})=\left(\operatorname{rk}\left(X_{1}\right)-\operatorname{rk}\left(X_{0}\right), \ldots, \operatorname{rk}\left(X_{d}\right)-\operatorname{rk}\left(X_{d-1}\right)\right) .
$$


Now we define

$$
\mathcal{G}[\mathbf{X}]=\sum_{\underline{X}} U_{r(\underline{X})}
$$

where $\underline{X}$ runs over all $d$ ! maximal chains in $X$. We will show that $\mathcal{G}[\mathbf{X}]$ behaves nicely with respect to direct sums and matroid duality. It defines a Hopf algebra homomorphism from the Hopf algebra of polymatroids to the Hopf algebra of quasi-symmetric functions. But unlike $\mathcal{F}[\mathbf{X}]$, it can distinguish between a loop and an isthmus. Moreover, $\mathcal{G}[\mathbf{X}]$ specializes to the Billera-Jia-Reiner quasi-symmetric function $\mathcal{F}[\mathbf{X}]$ as well as to $\mathcal{H}[\mathbf{X}](q, t)$. We will also show that $\mathcal{G}[\mathbf{X}]$ has the valuative property with respect to polymatroid polytope decompositions in Section 8. We question whether $\mathcal{G}[\mathbf{X}]$ might be universal with this property.

\section{Symmetric functions associated to polymatroids}

In this section we will define the invariants $\mathcal{H}[\mathbf{X}](q, t)$ and $\mathcal{P}[\mathbf{X}]$.

\subsection{Discrete polymatroids}

Definition 2.1 A (discrete) polymatroid is a pair $\mathbf{X}:=(X, \mathrm{rk})$ where $X$ is a finite set, and rk: $\operatorname{Pow}(X) \rightarrow \mathbb{N}=\{0,1,2, \ldots\}$ is a function satisfying

1. $\operatorname{rk}(\emptyset)=0$;

2. $\operatorname{rk}(A) \leq \operatorname{rk}(B)$ if $A \subseteq B$ (nondecreasing);

3. $\operatorname{rk}(A \cup B)+\operatorname{rk}(A \cap B) \leq \operatorname{rk}(A)+\operatorname{rk}(B)$ (submodular).

If $\mathbf{X}=(X, \mathrm{rk})$ is a polymatroid, and $A \subseteq X$ is a subset, then we restrict $\mathbf{X}$ to $A$ to get a polymatroid $\left.\mathbf{X}\right|_{A}:=\left(A,\left.\mathrm{rk}\right|_{A}\right)$. If $A^{\mathrm{c}}=X \backslash A$ is the complement, then the deletion of $A$ in $\mathbf{X}$ is the polymatroid $\mathbf{X} \backslash A:=\left.\mathbf{X}\right|_{A^{\mathrm{c}}}=\left(A^{\mathrm{c}},\left.\mathrm{rk}\right|_{A^{\mathrm{c}}}\right)$. The polymatroid $\mathbf{X} / A:=\left(A^{\mathrm{c}}, \mathrm{rk}_{X / A}\right)$ is defined by

$$
\operatorname{rk}_{X / A}(B)=\operatorname{rk}(A \cup B)-\operatorname{rk}(A)
$$

for all $B \subseteq A^{\mathrm{c}}$. We call $\mathbf{X} / A$ the contraction of $A$ in $\mathbf{X}$.

Two polymatroids $\mathbf{X}=\left(X, \mathrm{rk}_{X}\right)$ and $\mathbf{Y}=\left(Y, \mathrm{rk}_{Y}\right)$ are isomorphic if there exists a bijection $\varphi: X \rightarrow Y$ such that $\operatorname{rk}_{Y} \circ \varphi=\operatorname{rk}_{X}$. A polymatroid $\mathbf{X}=\left(X, \mathrm{rk}_{X}\right)$ is a matroid if $\operatorname{rk}_{X}(\{x\}) \in\{0,1\}$ for all $x \in X$. For more on matroids, see [30, 38]. If $\mathbf{X}=\left(X, \mathrm{rk}_{X}\right)$ is a matroid, then its dual is $\mathbf{X}^{\vee}:=\left(X, \mathrm{rk}_{X}^{\vee}\right)$ where $\operatorname{rk}_{X}^{\vee}$ is defined by

$$
\operatorname{rk}_{X}^{\vee}(A):=|A|-\operatorname{rk}_{X}(X)+\operatorname{rk}_{X}(X \backslash A)
$$

for all $A \subseteq X$. The Tutte polynomial behaves nicely with respect to matroid duality:

$$
\mathcal{T}\left[\mathbf{X}^{\vee}\right](x, y)=\mathcal{T}[\mathbf{X}](y, x) .
$$

There is also a formula expressing $\mathcal{F}\left[\mathbf{X}^{\vee}\right]$ in terms of $\mathcal{F}[\mathbf{X}]$ (see [3]). 
Definition 2.2 If $\mathbf{X}=\left(X, \mathrm{rk}_{X}\right)$ and $\mathbf{Y}=\left(Y, \mathrm{rk}_{Y}\right)$ are polymatroids, then we define their direct sum by

$$
\mathbf{X} \oplus \mathbf{Y}:=\left(X \sqcup Y, \mathrm{rk}_{X \sqcup Y}\right)
$$

where $X \sqcup Y$ is the disjoint union of $X$ and $Y$ and $\operatorname{rk}_{X \sqcup Y}: X \sqcup Y \rightarrow \mathbb{N}$ is defined by

$$
\operatorname{rk}_{X \sqcup Y}(A \cup B):=\operatorname{rk}_{X}(A)+\operatorname{rk}_{Y}(B)
$$

for all $A \subseteq X, B \subseteq Y$.

The Tutte polynomial satisfies the multiplicative property

$$
\mathcal{T}[\mathbf{X} \oplus \mathbf{Y}]=\mathcal{T}[\mathbf{X}] \cdot \mathcal{T}[\mathbf{Y}]
$$

2.2 The ring of symmetric functions

Let

$$
\text { Sym }:=\mathbb{Z}\left[e_{1}, e_{2}, e_{3}, \ldots\right] \subset \mathbb{Z}\left[x_{1}, x_{2}, x_{3}, \ldots\right]
$$

be the ring of symmetric functions in infinitely many variables, where

$$
e_{k}:=\sum_{i_{1}<i_{2}<\cdots<i_{k}} x_{i_{1}} x_{i_{2}} \cdots x_{i_{k}}
$$

is the $k$-th elementary symmetric function. The monomials in $e_{1}, e_{2}, \ldots$ form a $\mathbb{Z}$ basis of Sym. A partition of $n$ is a tuple $\lambda=\left(\lambda_{1}, \lambda_{2}, \ldots, \lambda_{r}\right)$ of positive integers with $\lambda_{1} \geq \cdots \geq \lambda_{r} \geq 1$ and $|\lambda|:=\lambda_{1}+\cdots+\lambda_{r}$ equal to $n$. Another basis of Sym is given by the Schur symmetric functions $s_{\lambda}$ where $\lambda$ runs over all partitions. For standard results on symmetric functions, we refer to the book [22]. The natural grading of $\mathbb{Z}\left[x_{1}, x_{2}, x_{3}, \ldots\right]$ induces a grading on $S y m$. In this grading $e_{k}$ has degree $k$ and $s_{\lambda}$ has degree $|\lambda|$. Let

$$
\overline{S y m}=\mathbb{Z}\left[\left[e_{1}, e_{2}, e_{3}, \ldots\right]\right]
$$

be the set of power series in $e_{1}, e_{2}, \ldots$ Define

$$
\sigma=1+s_{1}+s_{2}+s_{3}+\cdots \in \overline{\operatorname{Sym}} \text {. }
$$

The inverse is given by

$$
\sigma^{-1}=1-e_{1}+e_{2}-e_{3}+\cdots=1-s_{1}+s_{11}-s_{111}+\cdots .
$$

2.3 The definitions of $\mathcal{P}[\mathbf{X}]$ and $\mathcal{H}[\mathbf{X}](q, t)$

Definition 2.3 For every polymatroid $\mathbf{X}=(X, \mathrm{rk})$ we define a symmetric polynomial $\mathcal{P}[\mathbf{X}] \in$ Sym by induction as follows. If $X=\emptyset$, then $\mathcal{P}[\mathbf{X}]=1$. If $X \neq \emptyset$, then we may assume that $\mathcal{P}\left[\left.\mathbf{X}\right|_{A}\right]$ has been defined for all proper subsets $A \subset X$. We define

$$
\mathcal{P}[\mathbf{X}]=u_{0}+u_{1}+\cdots+u_{|X|-1}
$$


where $u_{i} \in$ Sym is homogeneous of degree $i$ for all $i$ such that

$$
\sum_{i=0}^{\infty} u_{i}=-\sum_{A \subset X} \mathcal{P}\left[\left.\mathbf{X}\right|_{A}\right] \sigma^{\mathrm{rk}(X)-\mathrm{rk}(A)}(-1)^{|X|-|A|} .
$$

Here $A$ runs over all proper subsets of $X$.

Definition 2.4 For every polymatroid $\mathbf{X}=(X, \mathrm{rk})$ we define a symmetric polynomial

$$
\mathcal{H}[\mathbf{X}](q, t) \in \operatorname{Sym}[q, t]=\mathbb{Z}[q, t] \otimes_{\mathbb{Z}} \operatorname{Sym}
$$

by

$$
\mathcal{H}[\mathbf{X}](q, t)=\sum_{A \subseteq X} \mathcal{P}\left[\left.\mathbf{X}\right|_{A}\right] q^{\mathrm{rk}(A)} t^{|A|}
$$

The coefficient of $t^{|X|}$ in $\mathcal{H}[\mathbf{X}](q, t)$ is $q^{\operatorname{rk}(X)} \mathcal{P}[\mathbf{X}]$.

Remark 2.5 If we evaluate (10) at $q=\sigma^{-1}$ and $t=-1$, then we obtain

$$
\mathcal{H}[\mathbf{X}]\left(\sigma^{-1},-1\right)=\sum_{A \subseteq X} \mathcal{P}\left[\left.\mathbf{X}\right|_{A}\right] \sigma^{-\operatorname{rk}(A)}(-1)^{|A|} \in \overline{S y m}
$$

From (8) and (9) it follows that $\mathcal{H}[\mathbf{X}]\left(\sigma^{-1},-1\right)$ vanishes in degree $<d=|X|$.

Proposition 2.6 (multiplicative property) For polymatroids $\mathbf{X}=\left(X, \mathrm{rk}_{X}\right)$ and $\mathbf{Y}=$ $\left(Y, \mathrm{rk}_{Y}\right)$ we have

$$
\mathcal{P}[\mathbf{X} \oplus \mathbf{Y}]=\mathcal{P}[\mathbf{X}] \cdot \mathcal{P}[\mathbf{Y}]
$$

and

$$
\mathcal{H}[\mathbf{X} \oplus \mathbf{Y}](q, t)=\mathcal{H}[\mathbf{X}](q, t) \cdot \mathcal{H}[\mathbf{Y}](q, t)
$$

Proof We prove the proposition by induction on $|X|+|Y|$. The case where $X=Y=$ $\emptyset$ is clear. So let us assume that $|X|+|Y|>0$. We may assume that

$$
\mathcal{P}\left[\left.\left.\mathbf{X}\right|_{A} \oplus \mathbf{Y}\right|_{B}\right]=\mathcal{P}\left[\left.\mathbf{X}\right|_{A}\right] \cdot \mathcal{P}\left[\left.\mathbf{Y}\right|_{B}\right]
$$

for all subsets $A \subseteq X$ and $B \subseteq Y$ such that $A \neq X$ or $B \neq Y$.

$$
\begin{aligned}
& \mathcal{H}[\mathbf{X} \oplus \mathbf{Y}](q, t)=\sum_{C \subseteq X \sqcup Y} \mathcal{P}\left[\left.(\mathbf{X} \oplus \mathbf{Y})\right|_{C}\right] q^{\mathrm{rk}_{X \sqcup Y}(C)} t^{|C|} \\
& =\sum_{A \subseteq X} \sum_{B \subseteq Y} \mathcal{P}\left[\left.\left.\mathbf{X}\right|_{A} \oplus \mathbf{Y}\right|_{B}\right] q^{\mathrm{rk}_{X}(A)+\mathrm{rk}_{Y}(B)} t^{|A|+|B|} \\
& =\sum_{A \subseteq X} \mathcal{P}\left[\left.\mathbf{X}\right|_{A}\right] q^{\mathrm{rk}_{X}(A)} t^{|A|} \cdot \sum_{B \subseteq Y} \mathcal{P}\left[\left.\mathbf{Y}\right|_{B}\right] q^{\mathrm{rk}_{Y}(B)} t^{|B|}
\end{aligned}
$$




$$
\begin{aligned}
& +(\mathcal{P}[\mathbf{X} \oplus \mathbf{Y}]-\mathcal{P}[\mathbf{X}] \mathcal{P}[\mathbf{Y}]) q^{\mathrm{rk}_{X}(X)+\mathrm{rk}_{Y}(Y)} t^{|X|+|Y|} \\
= & \mathcal{H}[\mathbf{X}](q, t) \cdot \mathcal{H}[\mathbf{Y}](q, t) \\
& +(\mathcal{P}[\mathbf{X} \oplus \mathbf{Y}]-\mathcal{P}[\mathbf{X}] \mathcal{P}[\mathbf{Y}]) q^{\operatorname{rk}_{X}(X)+\operatorname{rk}_{Y}(Y)} t^{|X|+|Y|}
\end{aligned}
$$

If we substitute $q=\sigma^{-1}$ and $t=-1$ we get

$$
\begin{aligned}
\mathcal{H} & {[\mathbf{X} \oplus \mathbf{Y}]\left(\sigma^{-1},-1\right)-\mathcal{H}[\mathbf{X}]\left(\sigma^{-1},-1\right) \cdot \mathcal{H}[\mathbf{Y}]\left(\sigma^{-1},-1\right) } \\
& =(-1)^{|X|+|Y|}(\mathcal{P}[\mathbf{X} \oplus \mathbf{Y}]-\mathcal{P}[\mathbf{X}] \cdot \mathcal{P}[\mathbf{Y}]) \sigma^{-\operatorname{rk}_{X}(X)-\operatorname{rk}_{Y}(Y)}
\end{aligned}
$$

The left-hand side has no terms in degree $<|X|+|Y|$ by Remark 2.5 and

$$
\mathcal{P}[\mathbf{X} \oplus \mathbf{Y}]-\mathcal{P}[\mathbf{X}]-\mathcal{P}[\mathbf{Y}]
$$

is a symmetric polynomial of degree $<|X|+|Y|$. It follows that

$$
\mathcal{P}[\mathbf{X} \oplus \mathbf{Y}]=\mathcal{P}[\mathbf{X}] \cdot \mathcal{P}[\mathbf{Y}]
$$

From (13) it follows that

$$
\mathcal{H}[\mathbf{X} \oplus \mathbf{Y}](q, t)=\mathcal{H}[\mathbf{X}](q, t) \cdot \mathcal{H}[\mathbf{Y}](q, t)
$$

The Tutte polynomial is closely related to the rank generating function

$$
\mathcal{R}[\mathbf{X}](q, t)=\sum_{A \subseteq X} q^{\mathrm{rk}(A)} t^{|A|} .
$$

We have

$$
(x-1)^{\operatorname{rk}(X)} \mathcal{R}[\mathbf{X}]\left((y-1)^{-1}(x-1)^{-1},(y-1)\right)=\mathcal{T}[\mathbf{X}](x, y),
$$

so the Tutte polynomial is completely determined by the rank generating function and vice versa. The rank generating function makes sense for polymatroids, not just matroids. The Tutte invariant may not be a polynomial for polymatroids, because we could have $\operatorname{rk}(A)>|A|$ for some subset $A \subseteq X$. Define

$$
\Theta: \operatorname{Sym} \rightarrow \mathbb{Q}
$$

by

$$
\Theta\left(s_{\lambda}\right)= \begin{cases}1 & \text { if } \lambda=() ; \\ 0 & \text { otherwise }\end{cases}
$$

Using base extension, we also get a $\mathbb{Q}(q, t)$-linear map

$$
\operatorname{Sym} \otimes_{\mathbb{Q}} \mathbb{Q}(q, t) \rightarrow \mathbb{Q}(q, t)
$$

which we also will denote by $\Theta$. It is straightforward to prove by induction on $|X|$ that $\Theta(\mathcal{P}[\mathbf{X}])=1$. 
Corollary 2.7 We have

$$
\Theta(\mathcal{H}[\mathbf{X}](q, t))=\sum_{A \subseteq X} q^{\mathrm{rk}(A)} t^{|A|}=\mathcal{R}[\mathbf{X}](q, t) .
$$

So $\mathcal{H}[\mathbf{X}](q, t)$ specializes to the rank generating function and the Tutte polynomial.

\section{Examples}

Example 3.1 Let $\mathbf{0}=\left(\{v\}, \mathrm{rk}_{\mathbf{0}}\right)$ be the loop matroid, and $\mathbf{1}=\left(\{v\}, \mathrm{rk}_{\mathbf{1}}\right)$ be the co-loop matroid defined by

$$
\operatorname{rk}_{\mathbf{0}}(v)=0 \text { and } r k_{1}(v)=1 .
$$

Then we have $P[\mathbf{0}]=P[\mathbf{1}]=1, \mathcal{H}[\mathbf{0}]=1+t, \mathcal{H}[\mathbf{1}]=1+q t, \mathcal{G}[\mathbf{0}]=U_{(0)}$ and $\mathcal{G}[\mathbf{1}]=U_{(1)}$.

An important class of matroids is the class of graphical matroids. Suppose that $\Gamma=$ $(Y, X, \phi)$ where $Y$ is the set of vertices, $X$ is the set of edges, and $\phi: X \rightarrow \operatorname{Pow}(Y)$ is a map such that $\phi(e)$ is the set of endpoints of the edge $e$. So $\phi(e)$ has 1 or 2 elements for all $e \in X$. Let $V=K^{n}$, and denote the coordinate functions by $x_{1}, \ldots, x_{n}$. To each vertex $e \in X$, with $\phi(e)=\{i, j\}$ we can associate a subspace $V_{e} \subseteq V$ defined by $x_{i}=x_{j}$. So $V_{e}$ is a hyperplane unless $e$ is a loop (i.e., $i=j$ ), in which case $V_{e}=V$. For $A \subseteq X$, we define $V_{A}=\bigcap_{a \in A} V_{a}$. We define a rank function by

$$
\operatorname{rk}(A)=\operatorname{dim} V-\operatorname{dim} V_{A}, \quad A \subseteq X .
$$

Now $\mathbf{X}=(X, \mathrm{rk})$ is a matroid.

Example 3.2 Suppose $(Y, X, \phi)$ is an $m$-gon.

$$
m=6 \text { : }
$$

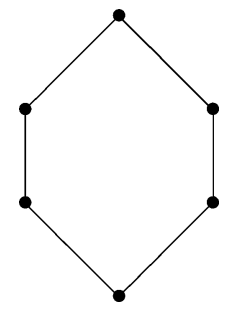

Then we have

$$
\begin{gathered}
\mathcal{T}[\mathbf{X}](x, y)=y+x+x^{2}+\cdots+x^{m-1}, \\
\mathcal{P}[\mathbf{X}]=1-s_{1}+s_{11}-\cdots+(-1)^{m-1} s_{1^{m-1}}, \\
\mathcal{H}[\mathbf{X}](q, t)=(1+q t)^{m}-(q t)^{m}+q^{m-1} t^{m} \mathcal{P}[\mathbf{X}],
\end{gathered}
$$




$$
\mathcal{G}[\mathbf{X}]=m ! U_{(1,1, \ldots, 1,0)}
$$

Example 3.3 Suppose that $(Y, X, \phi)$ is the graph with 2 vertices and $m$ edges between them.

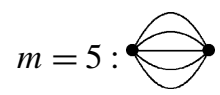

Then we have

$$
\begin{gathered}
\mathcal{T}[\mathbf{X}](x, y)=x+y+y^{2}+\cdots+y^{m-1}, \\
\mathcal{P}[\mathbf{X}]=1-\left(\begin{array}{c}
m-1 \\
1
\end{array}\right) s_{1}+\left(\begin{array}{c}
m-1 \\
2
\end{array}\right) s_{2}-\cdots+(-1)^{m-1}\left(\begin{array}{c}
m-1 \\
m-1
\end{array}\right) s_{m-1}, \\
\mathcal{H}[\mathbf{X}](q, t)=1+q \sum_{i=1}^{m}\left(\begin{array}{c}
m \\
i
\end{array}\right) t^{i}\left(\sum_{j=0}^{i-1}(-1)^{j}\left(\begin{array}{c}
i-1 \\
j
\end{array}\right) s_{j}\right) .
\end{gathered}
$$

Here, we use the convention $s_{0}=1$. To prove the formulas (14) and (15) it suffices to show that the right-hand side of (15) vanishes in degree $<m$ if we substitute $q=\sigma^{-1}$ and $t=-1$. If we make these substitutions, we get (using the combinatorial identity $[23, \S 1.2 .6,(33)])$

$$
\begin{aligned}
1 & +\sigma^{-1} \sum_{i=1}^{m}\left(\begin{array}{c}
m \\
i
\end{array}\right)(-1)^{i}\left(\sum_{j=0}^{i-1}(-1)^{j}\left(\begin{array}{c}
i-1 \\
j
\end{array}\right) s_{j}\right) \\
& =1+\sigma^{-1} \sum_{j=0}^{m-1} s_{j} \sum_{i=j+1}^{m}(-1)^{i+j}\left(\begin{array}{c}
m \\
i
\end{array}\right)\left(\begin{array}{c}
i-1 \\
j
\end{array}\right) \\
& =1+\sigma^{-1} \sum_{j=0}^{m-1} s_{j}\left((-1)^{j+1}\left(\begin{array}{c}
-1 \\
j
\end{array}\right)+\sum_{i=0}^{m}(-1)^{i+j}\left(\begin{array}{c}
m \\
i
\end{array}\right)\left(\begin{array}{c}
i-1 \\
j
\end{array}\right)\right) \\
& =1-\sigma^{-1} \sum_{j=0}^{m-1} s_{j} .
\end{aligned}
$$

This vanishes in degree $<m$ because $\sigma=1+s_{1}+s_{2}+\cdots$. We also have

$$
\mathcal{G}[\mathbf{X}]=m ! U_{(1,0,0, \ldots, 0)} .
$$

The following example appeared in [5], and was pointed out to the author by Nathan Reading. 
Example 3.4 The Gray graphs
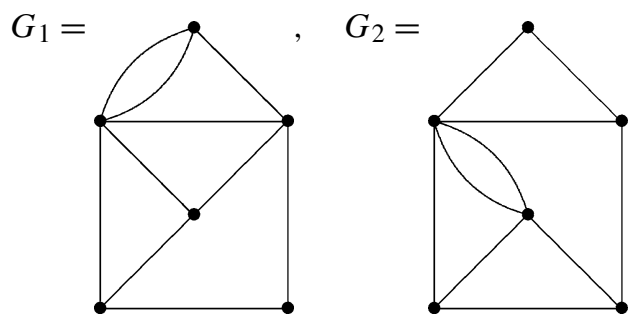

have the same Tutte polynomial, namely

$$
\begin{aligned}
\mathcal{T}\left[G_{1}\right](x, y)= & \mathcal{T}\left[G_{2}\right](x, y)=y^{5}+4 y^{4}+x y^{4}+x^{2} y^{3}+6 x y^{3}+7 y^{3} \\
& +x^{3} y^{2}+6 y^{2}+6 x^{2} y^{2}+13 x y^{2}+10 x y+x^{4} y+13 x^{2} y+6 x^{3} y \\
& +2 y+2 x+7 x^{3}+x^{5}+4 x^{4}+6 x^{2} .
\end{aligned}
$$

However, the coefficients of $s_{2,2,2}$ in $\mathcal{P}\left[G_{1}\right]$ and $\mathcal{P}\left[G_{2}\right]$ are 56 and 55 respectively.

The examples below appeared in the survey of Brylawski and Oxley in [39, pp. 197], and were also featured in [3].

Example 3.5 Consider 6 points in $\mathbb{P}^{2}=\mathbb{P}^{2}(\mathbb{C})$ according to the diagram below

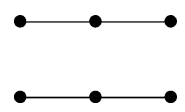

Here 3 or more points are collinear if and only if they lie on a line segment in the diagram. Dualizing gives us 6 projective lines in $\mathbb{P}^{2}$ which can be viewed as 6 hyperplanes in $\mathbb{C}^{3}$.

Denote the matroid associated with this arrangement by $\mathbf{X}$. Consider 6 points in $\mathbb{P}^{2}$ according to the diagram below

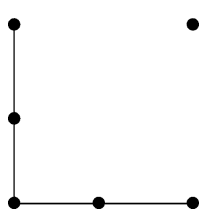

Again, dualizing gives a hyperplane arrangement in $\mathbb{C}^{3}$. Denote the matroid associated with this arrangement by $\mathbf{Y}$.

Then $\mathbf{X}$ and $\mathbf{Y}$ give nonisomorphic matroids, but they have the same Tutte polynomial and the same Billera-Jia-Reiner quasi-symmetric function (see [3]). Moreover,

$$
\begin{aligned}
\mathcal{P}[\mathbf{X}]=\mathcal{P}[\mathbf{Y}]= & 1-3 s_{1}+3 s_{2}+6 s_{1,1}-s_{3}-8 s_{2,1}-8 s_{1,1,1} \\
& +3 s_{3,1}+6 s_{2,2}+11 s_{2,1,1}-3 s_{3,2}-4 s_{3,1,1}-3 s_{2,2,1},
\end{aligned}
$$




$$
\mathcal{H}[\mathbf{X}](q, t)=\mathcal{H}[\mathbf{Y}](q, t)
$$

and

$$
\mathcal{G}[\mathbf{X}]=\mathcal{G}[\mathbf{Y}]=72 U_{(1,1,0,1,0,0)}+648 U_{(1,1,1,0,0,0)} .
$$

The last equation can easily be computed by hand as follows. There are 6 ! ways of labeling the points in diagram (17) by $p_{1}, p_{2}, p_{3}, p_{4}, p_{5}, p_{6}$. If $p_{1}, p_{2}, p_{3}$ are not colinear, then the labeling gives the rank sequence $(1,1,0,1,0,0)$, because $p_{1}$ spans a subspace of dimension 1 in $\mathbb{C}^{3}, p_{1}$ and $p_{2}$ span a subspace of dimension $1+1$, $p_{1}, p_{2}, p_{3}$ span a subspace of dimension $1+1+0, p_{1}, p_{2}, p_{3}, p_{4}$ span a subspace of dimension $1+1+0+1$, etc. There are $2 \cdot 3 !^{2}=72$ ways of choosing a labeling such that $p_{1}, p_{2}, p_{3}$ are colinear. All other $720-72=648$ labelings, give the rank sequence $(1,1,1,0,0,0)$. A similar reasoning can be used to compute $\mathcal{G}[\mathbf{Y}]$.

Example 3.6 Let $\mathbf{X}$ be the matroid corresponding to the hyperplane arrangement dual to the point arrangement of the following diagram

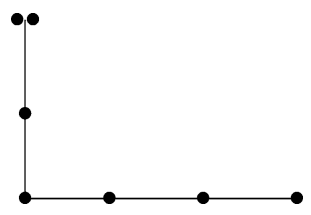

Let $\mathbf{Y}$ be the matroid corresponding to the hyperplane arrangement dual to the point arrangement of the following diagram

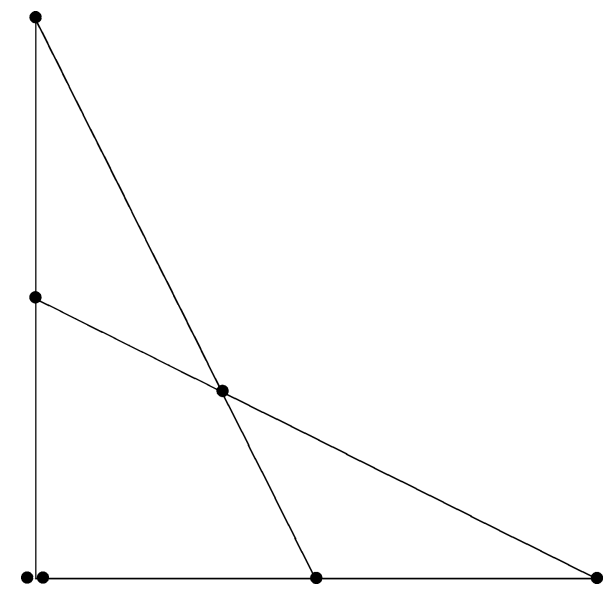

The Tutte polynomial is the same for $\mathbf{X}$ and $\mathbf{Y}$. The Billera-Jia-Reiner quasisymmetric function does distinguish the arrangements. We have

$$
\begin{aligned}
\mathcal{P}[\mathbf{X}]= & 1-4 s_{1}+6 s_{2}+9 s_{1,1}-4 s_{3}-17 s_{2,1}-10 s_{1,1,1} \\
& +s_{4}+12 s_{3,1}+13 s_{2,2}+17 s_{2,1,1}-3 s_{4,1}-10 s_{3,2}-10 s_{3,1,1}-8 s_{2,2,1} \\
& +2 s_{4,2}+2 s_{4,1,1}+2 s_{3,3}+3 s_{3,2,1}+s_{2,2,2}
\end{aligned}
$$


and

$$
\begin{aligned}
\mathcal{P}[\mathbf{Y}]= & 1-4 s_{1}+6 s_{2}+9 s_{1,1}-4 s_{3}-17 s_{2,1}-10 s_{1,1,1} \\
& +s_{4}+12 s_{3,1}+14 s_{2,2}+17 s_{2,1,1}-3 s_{4,1}-12 s_{3,2}-10 s_{3,1,1}-10 s_{2,2,1} \\
& +3 s_{4,2}+2 s_{4,1,1}+2 s_{3,3}+4 s_{3,2,1}+s_{2,2,2} .
\end{aligned}
$$

We also have

$$
\begin{aligned}
\mathcal{G}[\mathbf{X}]= & 3456 U_{(1,1,1,0,0,0,0)}+1080 U_{(1,1,0,1,0,0,0)}+264 U_{(1,1,0,0,1,0,0)} \\
& +216 U_{(1,0,1,1,0,0,0)}+24 U_{(1,0,1,0,1,0,0)}
\end{aligned}
$$

and

$$
\begin{aligned}
\mathcal{G}[\mathbf{Y}]= & 3456 U_{(1,1,1,0,0,0,0)}+1104 U_{(1,1,0,1,0,0,0)}+240 U_{(1,1,0,0,1,0,0)} \\
& +192 U_{(1,0,1,1,0,0,0)}+48 U_{(1,0,1,0,1,0,0)} .
\end{aligned}
$$

So the invariants $\mathcal{H}, \mathcal{P}$ and $\mathcal{G}$ distinguish these two matroids as well.

\section{Ideals and regularity}

\subsection{Equivariant free resolutions}

Let $K$ be a field, and $V$ be an $n$-dimensional $K$-vector space. For any partition $\lambda$, $S_{\lambda}$ denotes its corresponding Schur functor. In particular, $S_{d} V$ is the $d$-th symmetric power of $V$, and $S_{1^{d}} V=S_{1, \ldots, 1} V$ is the $d$-th exterior power. Let $R=K[V]$ be the ring of polynomial functions on $V$. The space $R_{d}$ of polynomial functions of degree $d$ can be identified with $S_{d}(Z)$, where $Z=V^{\star}$ is the dual space of $V$. Also, the ring $R=\bigoplus_{d=0}^{\infty} R_{d}$ can be identified with the symmetric algebra $S(Z):=\bigoplus_{d=0}^{\infty} S_{d}(Z)$ on $Z=V^{\star}$. By choosing a basis in $V$ and a dual basis $\left\{x_{1}, \ldots, x_{n}\right\}$ in $V^{\star}$ we may identify $R$ with the polynomial ring $K\left[x_{1}, \ldots, x_{n}\right]$. Let $\mathfrak{m}=\bigoplus_{d=1}^{\infty} R_{d}=\left(x_{1}, \ldots, x_{n}\right)$ be the maximal homogeneous ideal of $R$.

Suppose that $M$ is a finitely generated graded $R$-module. Its minimal resolution can be constructed as follows. First define $D_{0}:=M$ and $E_{0}=D_{0} / \mathfrak{m} D_{0}$. Then $E_{0}$ is a finite dimensional, graded vector space. The homogeneous quotient map $\psi_{0}: D_{0} \rightarrow$ $E_{0}$ has a homogeneous linear section $\phi_{0}: E_{0} \rightarrow D_{0}$ (which does not need to be an $R$-module homomorphism) such that $\psi_{0} \circ \phi_{0}=\mathrm{id}$. We can extend $\phi_{0}$ to a $R$-module homomorphism $\phi_{0}: R \otimes_{K} E_{0} \rightarrow D_{0}$ in a unique way. The tensor product $R \otimes_{K} E_{0}$ has a natural grading as a tensor product of two graded vector spaces, and $\phi_{0}$ is homogeneous with respect to this grading. We inductively define $D_{i}, E_{i}, \psi_{i}, \phi_{i}$ as follows. Define $D_{i}$ as the kernel of $\phi_{i-1}: R \otimes E_{i-1} \rightarrow D_{i-1}$. We set $E_{i}=D_{i} / \mathfrak{m} D_{i}$. Let $\phi_{i}: E_{i} \rightarrow D_{i}$ be a homogeneous linear section to the homogeneous quotient map $\psi_{i}: D_{i} \rightarrow E_{i}$. We can extend $\phi_{i}$ to an $R$-module homomorphism $\phi_{i}: R \otimes E_{i} \rightarrow D_{i}$. By Hilbert's Syzygy theorem (see [21] and [13, Corollary 19.7]), we get that $D_{i}=0$ for $i>n$. We end up with the minimal free resolution

$$
0 \rightarrow R \otimes E_{n} \rightarrow R \otimes E_{n-1} \rightarrow \cdots R \otimes E_{0} \rightarrow M \rightarrow 0 .
$$


Here $E_{i}$ can be naturally identified with $\operatorname{Tor}_{j}(M, K)$.

For a group $G$ and sets $X$ and $Y$ on which $G$ acts, we say that a map $\phi: X \rightarrow Y$ is $G$-equivariant if it respects the action, i.e., $\phi(g \cdot x)=g \cdot \phi(x)$ for all $x \in X$ and $g \in G$. Suppose that $G$ is a linearly reductive linear algebraic group and $V$ is a representation of $G$. Assume that $G$ also acts on the finitely generated graded $R$-module $M=\bigoplus_{d} M_{d}$ such the multiplication $R \times M \rightarrow M$ is $G$-equivariant, and $M_{d}$ is a representation of $G$ for every $d$. By the definition of linear reductivity, we can choose the sections $\phi_{i}: E_{i} \rightarrow K_{i}$ to be $G$-equivariant. So by induction we see that $G$ acts regularly on $D_{0}, E_{0}, D_{1}, E_{1}, D_{2}, E_{2}, \ldots$ Also, by induction one can show that the structure of $D_{i}$ as a $G$-equivariant graded $R$-module, and $E_{i}$ as graded representation of $G$ do not depend on the choices of the $G$-equivariant sections $\phi_{i}$. We conclude that $E_{i} \cong \operatorname{Tor}_{i}(M, K)$ has a well-defined structure as a graded $G$-module.

\subsection{Castelnuovo-Mumford regularity}

For a finite dimensional graded $K$-vector space $W=\bigoplus_{d \in \mathbb{Z}} W_{d}$ we define

$$
\operatorname{deg}(W):=\max \left\{i \mid W_{i} \neq 0\right\} .
$$

If $W=\{0\}$ then we define $\operatorname{deg}(W)=-\infty$. A finitely generated graded $R$-module $M$ is called $s$-regular if $\operatorname{deg}\left(\operatorname{Tor}^{i}(M, K)\right) \leq s+i$ for all $i$. The Castelnuovo-Mumford regularity $\operatorname{reg}(M)$ of $M$ is the smallest integer $s$ such that $M$ is $s$-regular. See [13, $\S 20.5]$ for more on Castelnuovo-Mumford regularity.

\subsection{Product ideals and regularity bounds}

Suppose that $V_{x}, x \in X$ are subspaces of $V$ for some finite set $X$ with $d$ elements. Assume that $X=\{1,2, \ldots, d\}$. Let $J_{x} \subseteq K[V]=S(Z)$ be the vanishing ideal of $V_{x}$. The ideal $J_{x}$ is generated by the subspace $Z_{x}=V_{x}^{\perp} \subseteq Z=V^{\star}$ of all linear functions vanishing on $V_{x}$. For every subset $A \subseteq X$, we define $J_{A}:=\prod_{x \in X} J_{x}$, and let $J=J_{X}$. A crucial result we need is:

Theorem 4.1 (Conca and Herzog [7]) The Castelnuovo-Mumford regularity of $J$ is equal to $d$.

We define

$$
C_{k}=\bigoplus_{|A|=k} J_{A} .
$$

Following [33, Chapter IV] we construct a complex

$$
0 \rightarrow C_{d} \rightarrow C_{d-1} \rightarrow \cdots \rightarrow C_{0} \rightarrow 0
$$

The map $\partial_{k}: C_{k} \rightarrow C_{k-1}$ can be written as $\partial_{k}=\sum_{A, B} \partial_{k}^{A, B}$, where

$$
\partial_{k}^{A, B}: J_{A} \rightarrow J_{B}
$$


Suppose that $A=\left\{i_{1}, i_{2}, \ldots, i_{k}\right\}$ with $i_{1}<i_{2}<\cdots<i_{k}$, then we define

$$
\partial_{k}^{A, B}:= \begin{cases}0 & \text { if } B \nsubseteq A ; \\ (-1)^{r} \text { id } & \text { if } B=\left\{i_{1}, \ldots, i_{r-1}, i_{r+1}, \ldots, i_{k}\right\} .\end{cases}
$$

The homology of the complex is denoted by

$$
H_{k}=\operatorname{ker} \partial_{k} / \operatorname{im} \partial_{k+1} \text {. }
$$

Remark 4.2 Since $\partial_{d}$ is injective, we have that $H_{d}=0$.

Proposition 4.3 [33] If $V_{X}:=\bigcap_{x \in X} V_{x}=(0)$, then the homogeneous maximal ideal $\mathfrak{m}$ kills all homology, i.e., $\mathfrak{m} H_{i}=0$ for all $i$.

The following result is Corollary 20.19 in [13].

Lemma 4.4 If $A, B, C$ are finitely generated graded modules, and

$$
0 \rightarrow A \rightarrow B \rightarrow C \rightarrow 0
$$

is exact, then

1. $\operatorname{reg}(A) \leq \max \{\operatorname{reg}(B), \operatorname{reg}(C)+1\}$;

2. $\operatorname{reg}(B) \leq \max \{\operatorname{reg}(A), \operatorname{reg}(C)\}$;

3. $\operatorname{reg}(C) \leq \max \{\operatorname{reg}(A)-1, \operatorname{reg}(B)\}$.

Proposition 4.5 Suppose that $V_{X}=\bigcap_{x \in X} V_{x}=(0)$. Then $H_{k}$ is concentrated in degree $k$ (and in particular, it is finite dimensional).

Proof We have $\operatorname{reg}\left(C_{i}\right) \leq i$ by Theorem 4.1. Let $Z_{i}$ and $B_{i}$ be the kernel, respectively, the cokernel of $\partial_{i}$.

First, we prove that

$$
\operatorname{reg}\left(H_{i}\right) \leq \operatorname{reg}\left(B_{i}\right)-1
$$

for $i=0,1, \ldots, d-1$. Since $\mathfrak{m} H_{i}=0, H_{i}$ is just equal to a number of copies of $K$ in various degrees. From the Koszul resolution it follows that

$$
\operatorname{deg}\left(\operatorname{Tor}_{j}\left(H_{i}, K\right)\right)=\operatorname{deg}\left(H_{i}\right)+j
$$

for $j=0,1,2, \ldots, n$, hence $\operatorname{reg}\left(H_{i}\right)=\operatorname{deg}\left(H_{i}\right)$. The exact sequence

$$
0 \rightarrow B_{i} \rightarrow Z_{i} \rightarrow H_{i} \rightarrow 0
$$

gives rise to a long exact Tor sequence

$$
0 \rightarrow \operatorname{Tor}_{n}\left(B_{i}, K\right) \rightarrow \operatorname{Tor}_{n}\left(Z_{i}, K\right) \rightarrow \operatorname{Tor}_{n}\left(H_{i}, K\right) \rightarrow \operatorname{Tor}_{n-1}\left(B_{i}, K\right) \rightarrow \cdots .
$$

Since $Z_{i}$ is a submodule of a free module, its projective dimension is $\leq n-1$ and $\operatorname{Tor}_{n}\left(Z_{i}, K\right)=0$. Therefore

$$
\operatorname{deg}\left(\operatorname{Tor}_{n-1}\left(B_{i}, K\right)\right) \geq \operatorname{deg}\left(\operatorname{Tor}_{n}\left(H_{i}, K\right)\right)=\operatorname{reg}\left(H_{i}\right)+n .
$$


It follows that

$$
\operatorname{reg}\left(B_{i}\right)+n-1 \geq \operatorname{deg}\left(\operatorname{Tor}_{n-1}\left(B_{i}, K\right)\right) \geq \operatorname{reg}\left(H_{i}\right)+n .
$$

This proves (21).

From (22) and Lemma 4.4 it follows that

$$
\operatorname{reg}\left(Z_{i}\right) \leq \max \left\{\operatorname{reg}\left(B_{i}\right), \operatorname{reg}\left(H_{i}\right)\right\}=\operatorname{reg}\left(B_{i}\right) .
$$

By induction on $i$ we will show that $\operatorname{reg}\left(B_{d-i}\right) \leq d-i+1, \operatorname{reg}\left(Z_{d-i}\right) \leq d-i+1$ and $\operatorname{reg}\left(H_{d-i}\right) \leq d-i$. For $i=1$ we have $\operatorname{reg}\left(B_{d-1}\right)=\operatorname{reg}\left(C_{d}\right)=d, \operatorname{reg}\left(Z_{d-1}\right) \leq d$ by (23) and $\operatorname{reg}\left(H_{d-1}\right) \leq d-1$ by (21).

Suppose that $i>1$. We may assume by induction that $Z_{d-i+1}$ is $(d-i+2)$ regular. From the exact sequence

$$
0 \rightarrow Z_{d-i+1} \rightarrow C_{d-i+1} \rightarrow B_{d-i} \rightarrow 0
$$

it follows that

$$
\operatorname{reg}\left(B_{d-i}\right) \leq \max \left\{\operatorname{reg}\left(Z_{d-i+1}\right)-1, \operatorname{reg}\left(C_{d-i+1}\right)\right\} \leq d-i+1
$$

by Lemma 4.4. Now we have $\operatorname{reg}\left(Z_{d-i}\right) \leq d-i+1$ by (23) and $\operatorname{reg}\left(H_{d-i}\right) \leq d-i$ by (21).

Suppose that $G$ is a linearly reductive group and let $\widehat{G}$ denote the set of isomorphism classes of irreducible representations of $G$. Let $\mathbb{Z}^{\widehat{G}}$ be the set of maps $\widehat{G} \rightarrow \mathbb{Z}$. Elements of $\mathbb{Z}^{\widehat{G}}$ may be thought of as $G$-Hilbert series. If $M$ is a $G$-module such that every irreducible representation appears only finitely many times, then we define

$$
\langle M\rangle=\langle M\rangle_{G} \in \mathbb{Z}^{\widehat{G}} .
$$

For every irreducible represention $U$ of $G,\langle M\rangle(U)$ is the multiplicity of $U$ in $M$.

Lemma 4.6 Suppose that $G$ acts on $Z$ such that every irreducible representation of $G$ appears only finitely many times in $S(Z)$. Then we have

$$
\sum_{A \subset X}(-1)^{|A|}\left\langle J_{A}\right\rangle=\sum_{i=0}^{d}(-1)^{i}\left\langle C_{i}\right\rangle=\sum_{i=0}^{d-1}(-1)^{i}\left\langle H_{i}\right\rangle .
$$

Proof The first equality follows from the definition (19). For every $i$ we have exact sequences

$$
0 \rightarrow Z_{i} \rightarrow C_{i} \rightarrow \operatorname{im~} B_{i-1} \rightarrow 0
$$

and

$$
0 \rightarrow B_{i} \rightarrow Z_{i} \rightarrow H_{i} \rightarrow 0
$$


So we have

$$
\begin{aligned}
\sum_{i}(-1)^{i}\left\langle C_{i}\right\rangle & =\sum_{i}(-1)^{i}\left\langle Z_{i}\right\rangle+\sum_{i}(-1)^{i}\left\langle B_{i-1}\right\rangle \\
& =\sum_{i}(-1)^{i}\left\langle Z_{i}\right\rangle-\sum_{i}(-1)^{i}\left\langle B_{i}\right\rangle=\sum_{i}(-1)^{i}\left\langle H_{i}\right\rangle .
\end{aligned}
$$

\section{Realizable polymatroids}

\subsection{The tensor trick}

Let us fix a field $K$.

Definition 5.1 An arrangement realization of a polymatroid $\mathbf{X}=(X, \mathrm{rk})$ over $K$ is a finite dimensional $K$-vector space $V$ together with a collection of subspaces $V_{x}$, $x \in X$ such that

$$
\operatorname{rk}(A)=\operatorname{dim} V-\operatorname{dim} V_{A}
$$

for every $A \subseteq X$, where

$$
V_{A}=\bigcap_{x \in X} V_{x}
$$

Let $\mathbf{X}=(X, \mathrm{rk})$ be a polymatroid and set $d=|X|$. From now on, assume that $K$ is a field of characteristic 0. Suppose that $V$ is an $n$-dimensional $K$-vector space and $V_{x}$, $x \in X$ is a collection of subspaces that form a realization of $\mathbf{X}$.

Let $W$ be another $K$-vector space and let $R(W):=K\left[V \otimes W^{\star}\right]$ be the ring of polynomial functions on $V \otimes W^{\star}=\operatorname{Hom}(W, V)$. Note that $\operatorname{GL}(W)$ acts regularly on $K\left[V \otimes W^{\star}\right]$. Let $J_{x}(W) \subseteq R(W)$ be the vanishing ideal of $V_{x} \otimes W^{\star} \subseteq V \otimes W^{\star}$. For a subset $A \subseteq X$ we define

$$
J_{A}(W)=\prod_{x \in A} J_{x}(W)
$$

and we set $J(W):=J_{X}(W)$. Define

$$
C_{i}(W):=\bigoplus_{\substack{A \subseteq X \\|A|=i}} J_{A}(W)
$$

As in (20), we have a complex

$$
0 \rightarrow C_{d}(W) \rightarrow C_{d-1}(W) \rightarrow \cdots \rightarrow C_{1}(W) \rightarrow C_{0}(W) \rightarrow 0 .
$$

Let $H_{i}(W)$ be the $i$-th homology group. By Lemma 4.6, we have

$$
\sum_{i=0}^{d-1}(-1)^{i}\left\langle H_{i}(W)\right\rangle=\sum_{i=0}^{d}(-1)^{i}\left\langle C_{i}(W)\right\rangle=\sum_{A \subseteq X}(-1)^{|A|}\left\langle J_{A}(W)\right\rangle .
$$


If $f=\sum_{\lambda} a_{\lambda} s_{\lambda} \in \mathbb{Z}\left[\left[e_{1}, e_{2}, \ldots\right]\right]$, then we define

$$
f \star W=\sum a_{\lambda}\left\langle S_{\lambda}(W)\right\rangle
$$

For example, we have

$$
\sigma \star W=\left(s_{0}+s_{1}+s_{2}+s_{3}+\cdots\right) \star W=\sum_{i=0}^{\infty}\left\langle S_{i}(W)\right\rangle=\langle S(W)\rangle .
$$

If $f, g \in \mathbb{Z}\left[\left[e_{1}, e_{2}, \ldots\right]\right]$, then

$$
(f \cdot g) \star W=(f \star W) \otimes(g \star W) .
$$

5.2 Product ideals and the invariants $\mathcal{P}[\mathbf{X}], \mathcal{H}[\mathbf{X}](q, t)$

Theorem 5.2 We have

$$
\left(\sigma^{n-\operatorname{rk}(X)} \mathcal{P}[\mathbf{X}]\right) \star W=\sum_{A \subseteq X}(-1)^{|A|}\left\langle J_{A}(W)\right\rangle
$$

and

$$
\left(\sigma^{n} \mathcal{H}[X]\left(\sigma^{-1},-1\right)\right) \star W=\langle J(W)\rangle
$$

Proof We prove the statement by induction on $d=|X|$. If $X=\emptyset$, then $\mathcal{P}[\mathbf{X}]=1$ and

$$
\sigma^{n} \star W=\left\langle S(W)^{\otimes n}\right\rangle=\left\langle S\left(W \otimes V^{\star}\right)\right\rangle=\left\langle K\left[V \otimes W^{\star}\right]\right\rangle=\langle R(W)\rangle=\left\langle J_{\emptyset}(W)\right\rangle,
$$

so (28) holds.

For every $A \subseteq X$, define

$$
Z_{A}:=\sum_{B \subseteq A}(-1)^{|B|}\left\langle J_{B}(W)\right\rangle
$$

By Möbius inversion, we get

$$
\left\langle J_{B}(W)\right\rangle=\sum_{A \subseteq B}(-1)^{|A|} Z_{A} .
$$

By induction we may assume that

$$
\left(\sigma^{n-\operatorname{rk}(A)} \mathcal{P}\left[\left.\mathbf{X}\right|_{A}\right]\right) \star W=Z_{A}
$$

for all proper subsets $A \subset X$.

Let us assume that $V_{X}=(0)$. From (27) and Proposition 4.5 it follows that $Z_{X}$ is a combination of $\left\langle S_{\lambda}(W)\right\rangle$ with $|\lambda|<d$. Consider

$$
\left(\sigma^{n} \mathcal{H}[X]\left(\sigma^{-1},-1\right)\right) \star W-\langle J(W)\rangle
$$




$$
\begin{aligned}
& =\sum_{A \subseteq X}(-1)^{|A|}\left(\sigma^{n-\operatorname{rk}(A)} \mathcal{P}\left[\left.\mathbf{X}\right|_{A}\right]\right) \star W-\langle J(W)\rangle \\
& =(-1)^{|X|}\left(\sigma^{n-\mathrm{rk}(X)} \mathcal{P}[\mathbf{X}] \star W-Z_{X}\right)+\sum_{A \subseteq X}(-1)^{|A|} Z_{A}-\langle J(W)\rangle \\
& =(-1)^{|X|}\left(\sigma^{n-\mathrm{rk}(X)} \mathcal{P}[\mathbf{X}] \star W-Z_{X}\right) .
\end{aligned}
$$

In $\left(\sigma^{n} \mathcal{H}[X]\left(\sigma^{-1},-1\right)\right) \star W$ and $\langle J(W)\rangle$ only terms $\left\langle S_{\lambda}(W)\right\rangle$ appear with $|\lambda| \geq d$. On the other hand, in $\sigma^{n-\operatorname{rk}(X)} \mathcal{P}[\mathbf{X}] \star W$ and $Z_{X}$ only terms $\left\langle S_{\lambda}(W)\right\rangle$ appear with $|\lambda|<d$. It follows that the left-hand side and the right-hand side of (30) are equal to 0 .

Suppose that $V_{X} \neq(0)$. Let $V^{\prime}$ be a complement of $V_{X}$ in $V$ of dimension $n-$ $r(X)$. Define $V_{x}^{\prime}=V^{\prime} \cap V_{x}$ for all $x \in X$ and $V_{A}^{\prime}=V^{\prime} \cap V_{A}=\bigcap_{x \in A} V_{x}^{\prime}$ for all $A \subseteq X$. We have that $V_{X}^{\prime}=V^{\prime} \cap V_{X}=(0)$ and $V_{A}^{\prime}=V_{A}^{\prime} \oplus V_{X}$ for all $A \subseteq X$. It follows that

$$
\begin{aligned}
\operatorname{rk}(A)=\operatorname{dim} V-\operatorname{dim} V_{A} & =\left(\operatorname{dim} V^{\prime}+\operatorname{dim} V_{X}\right)-\left(\operatorname{dim} V_{A}^{\prime}+\operatorname{dim} V_{X}\right) \\
& =\operatorname{dim} V^{\prime}-\operatorname{dim} V_{A}^{\prime} .
\end{aligned}
$$

Let $J_{x}^{\prime}(W) \subseteq K\left[V^{\prime} \otimes W^{\star}\right]$ be the vanishing ideal of $V_{x}^{\prime} \otimes W^{\star}$ inside $V^{\prime} \otimes W^{\star}$. Define $J_{A}^{\prime}(W)=\prod_{x \in A} J_{x}^{\prime}(W)$ and set $J^{\prime}(W)=J_{X}^{\prime}(W)$. By the previous case,

$$
\left(\sigma^{\mathrm{rk}(X)} \mathcal{H}[X]\left(\sigma^{-1},-1\right)\right) \star W=\left\langle J^{\prime}(W)\right\rangle
$$

and

$$
\mathcal{P}[\mathbf{X}] \star W=\sum_{A \subseteq X}(-1)^{|A|}\left\langle J_{A}^{\prime}(W)\right\rangle .
$$

It follows that

$$
J(W)=J^{\prime}(W) \otimes S\left(V_{X}^{\star} \otimes W\right)=J^{\prime}(W) \otimes S(W)^{\otimes(n-\mathrm{rk}(X))}
$$

and

$$
\begin{aligned}
\left(\sigma^{n} \mathcal{H}[X]\left(\sigma^{-1},-1\right)\right) \star W & =\left(\sigma^{\operatorname{rk}(X)} \mathcal{H}[X]\left(\sigma^{-1},-1\right)\right) \star W \otimes\left\langle S(W)^{\otimes(n-\operatorname{rk}(X))}\right\rangle \\
& =\left\langle J^{\prime}(W) \otimes S(W)^{\otimes(n-\operatorname{rk}(X))}\right\rangle=\langle J(W)\rangle .
\end{aligned}
$$

Similarly, from

$$
\mathcal{P}[\mathbf{X}] \star W=\sum_{A \subseteq X}(-1)^{|A|}\left\langle J_{A}^{\prime}(W)\right\rangle
$$

it follows that

$$
\begin{aligned}
\left(\sigma^{n-\mathrm{rk}(X)} \mathcal{P}[\mathbf{X}]\right) \star W & =\sum_{A \subseteq X}(-1)^{|A|}\left\langle J_{A}^{\prime}(W) \otimes S(W)^{\otimes(n-\mathrm{rk}(X))}\right\rangle \\
& =\sum_{A \subseteq X}(-1)^{|A|}\left\langle J_{A}(W)\right\rangle .
\end{aligned}
$$


Corollary 5.3 Suppose that $V_{X}=(0)$. If we write

$$
\mathcal{P}[\mathbf{X}]=u_{0}-u_{1}+u_{2}-\cdots+(-1)^{d-1} u_{d-1}
$$

where $u_{i}$ is a homogeneous symmetric polynomial of degree $i$ for all $i$, then

$$
u_{i} \star W=\left\langle H_{i}(W)\right\rangle \text {. }
$$

Proposition 5.4 We can write

$$
\mathcal{H}[\mathbf{X}]\left(\sigma^{-1},-1\right)=w_{d}-w_{d+1}+w_{d+2}-w_{d+3}+\cdots,
$$

where $d=|X|$ and $w_{i}$ is a homogeneous symmetric polynomial of degree $i$. We have

$$
w_{d+i} \star W=\left\langle\operatorname{Tor}_{i}(J(W), K)\right\rangle \text {. }
$$

Proof Since $J(W)$ is $d$-regular and generated in degree $d$, it has a linear minimal free resolution. We can choose this resolution to be $\mathrm{GL}(W)$-equivariant. Define

$$
E_{i}(W):=\operatorname{Tor}_{i}(J(W), K) .
$$

The minimal resolution has the form

$0 \rightarrow E_{\ell}(W) \otimes R(W) \rightarrow \cdots \rightarrow E_{1}(W) \otimes R(W) \rightarrow E_{0}(W) \otimes R(W) \rightarrow J(W) \rightarrow 0$,

where $\ell=\operatorname{pd}(J(W))$ is the projective dimension of $J(W)$. We have

$$
\left(\sigma^{n} \mathcal{H}[\mathbf{X}]\left(\sigma^{-1},-1\right)\right) \star W=\langle J(W)\rangle=\sum_{i=0}^{\ell}(-1)^{i}\left\langle E_{i}(W) \otimes R(W)\right\rangle,
$$

so

$$
\mathcal{H}[\mathbf{X}]\left(\sigma^{-1},-1\right) \star W=\left(\sum_{i=0}^{\infty}(-1)^{i} w_{d+i}\right) \star W=\sum_{i=0}^{\ell}(-1)^{i}\left\langle E_{i}(W)\right\rangle .
$$

Example 5.5 Let $V=\mathbb{C}$ and let $V_{1}=V_{2}=\cdots=V_{d}=\{0\}$. The rank function is the same as in Example 3.3.

$$
\mathcal{H}[\mathbf{X}](q, t)=1+q \sum_{i=1}^{d}\left(\begin{array}{l}
d \\
i
\end{array}\right) t^{i}\left(\sum_{j=0}^{i-1}(-1)^{j}\left(\begin{array}{c}
i-1 \\
j
\end{array}\right) s_{j}\right) .
$$

The ideal $J(W)=\mathfrak{m}(W)^{d}$ where $\mathfrak{m}(W)$ is the maximal homogeneous ideal in $K[V \otimes$ $\left.W^{\star}\right] \cong K\left[W^{\star}\right] \cong S(W)$.

For $d=1$ we have

$$
\mathcal{H}[\mathbf{X}](q, t)=1+q t
$$

It follows that

$$
\mathcal{H}[\mathbf{X}]\left(\sigma^{-1},-1\right)=1-\sigma^{-1}=s_{1}-s_{1,1}+s_{1,1,1}-\cdots .
$$


This shows that the $i$-th free module in the free resolution is $S(W) \otimes S_{1,1, \ldots, 1} W \cong$ $S(W) \otimes \bigwedge^{i}(W)$. So the minimal resolution is

$$
\cdots \rightarrow S(W) \otimes S_{1,1}(W) \rightarrow S(W) \otimes W \rightarrow \mathfrak{m}(W) \rightarrow 0,
$$

which is of course the Koszul resolution of the maximal ideal $\mathfrak{m}(W)$. For $d=2$, we get

$$
\mathcal{H}[\mathbf{X}](q, t)=1+2 q t+q t^{2}\left(1-s_{1}\right)
$$

and

$$
\mathcal{H}[\mathbf{X}]\left(\sigma^{-1},-1\right)=1-\sigma^{-1}\left(1+s_{1}\right)=s_{2}-s_{2,1}+s_{2,1,1}-\cdots .
$$

So this means the equivariant minimal free resolution of $\mathfrak{m}(W)^{2}$ looks like

$$
\cdots \rightarrow S(W) \otimes S_{2,1,1}(W) \rightarrow S(W) \otimes S_{2,1}(W) \rightarrow S(W) \otimes S_{2}(W) \rightarrow \mathfrak{m}(W)^{2} \rightarrow 0 .
$$

5.3 Nonnegativity results for the coefficients of $\mathcal{P}[\mathbf{X}]$ and $\mathcal{H}[\mathbf{X}](q, t)$

Corollary 5.6 Suppose that $\mathbf{X}=(X, \mathrm{rk})$ is realizable over a field $K$ of characteristic 0 .

1.

$$
\sigma^{\mathrm{rk}(X)} \mathcal{H}[\mathbf{X}]\left(\sigma^{-1},-1\right)=\sum_{\lambda} a_{\lambda} s_{\lambda}
$$
2 .

where $\lambda$ runs over all partitions with $|\lambda| \geq d$ and $a_{\lambda} \geq 0$ for all $\lambda$;

$$
\mathcal{P}[\mathbf{X}]=\sum_{\lambda}(-1)^{|\lambda|} b_{\lambda} s_{\lambda},
$$

where $\lambda$ runs over all partitions with $|\lambda|<d$ and $b_{\lambda} \geq 0$ for all $\lambda$;

3.

$$
\mathcal{H}[\mathbf{X}]\left(\sigma^{-1},-1\right)=\sum_{\lambda}(-1)^{|\lambda|} c_{\lambda} s_{\lambda},
$$

where $\lambda$ runs over all partitions $\lambda$ with $|\lambda| \geq d$ with more than $|\lambda| / \operatorname{rk}(X)$ parts, and $c_{\lambda} \geq 0$ for all $\lambda$.

Proof Assume, as before, that $V$ together with $V_{x}, x \in X$ form a realization of $\mathbf{X}$. We may also assume that $V_{X}=(0)$.

(1) From Remark 2.5 it follows that no $s_{\lambda}$ with $|\lambda|<d$ appears in the left-hand side of (32). If we choose $\operatorname{dim} W \geq|\lambda|$ then $S_{\lambda}(W) \neq 0$ and $\left\langle S_{\lambda}(W)\right\rangle$ appears with a nonnegative coefficient on the right-hand side of (31). Therefore, the coefficient of $s_{\lambda}$ in $\sigma^{\operatorname{rk}(X)} \mathcal{H}[\mathbf{X}]\left(\sigma^{-1},-1\right)$ is nonnegative.

(2) This follows from Corollary 5.3.

(3) The nonnegativity of $c_{\lambda}$ follows from Proposition 5.4. If $\ell=\operatorname{pd}(J(W))$ is the projective dimension of $J(W)$, then we have

$$
\ell=\operatorname{pd}(J(W))=\operatorname{pd}(R(W) / J(W))-1<\operatorname{dim} V \operatorname{dim} W=\operatorname{rk}(X) \operatorname{dim} W .
$$


Suppose $\lambda=\left(\lambda_{1}, \ldots, \lambda_{k}\right)$ and the coefficient of $s_{\lambda}$ in $\mathcal{H}[\mathbf{X}]\left(\sigma^{-1},-1\right)$ is nonzero. If $W$ is $k$-dimensional, then $S_{\lambda}(W) \neq 0$, so $E^{|\lambda|}(W) \neq 0$ and $|\lambda| \leq \ell<\operatorname{rk}(X) k$.

Conjecture 5.7 Corollary 5.6 is true, even if $\mathbf{X}=(X, \mathrm{rk})$ is a polymatroid that is not realizable.

5.4 The Rees ring and the invariant $\tilde{H}[\mathbf{X}](q, t, y)$

Instead of looking at the GL(W)-Hilbert series of $J(W)$, one could also consider the $\mathrm{GL}(W)$-Hilbert series of the Rees ring

$$
R(W)[y J(W)]=R(W) \oplus y J(W) \oplus y^{2} J(W)^{2} \oplus \cdots,
$$

where $y$ is an indeterminate. This Hilbert series is

$$
\sigma^{n} \sum_{i=0}^{\infty} \mathcal{H}\left[\mathbf{X}^{i}\right]\left(\sigma^{-1},-1\right) y^{i}
$$

where

$$
\mathbf{X}^{i}=\underbrace{\mathbf{X} \oplus \mathbf{X} \oplus \cdots \oplus \mathbf{X}}_{i} .
$$

It is therefore natural to define the invariant

$$
\tilde{\mathcal{H}}[\mathbf{X}](q, t, y):=\sum_{i=0}^{\infty} \mathcal{H}\left[\mathbf{X}^{i}\right](q, t) y^{i} .
$$

Another interesting ring is the subalgebra $T(W)$ of $R(W)$ generated by

$$
\left(W \otimes Z_{1}\right)\left(W \otimes Z_{2}\right) \cdots\left(W \otimes Z_{d}\right) .
$$

The degree $k d$ part in $T(W)$ (or degree $k$ after rescaling) is equal to the degree $(k d, d)$ part in $R(W)$. If we take

$$
\sigma^{n} \tilde{\mathcal{H}}[\mathbf{X}]\left(\sigma^{-1},-1, z^{-1}\right),
$$

replace $s_{\lambda}$ by $z^{|\lambda| d} s_{\lambda}$ for all $\lambda$ and then set $z=0$, then we obtain the Hilbert series of $T(W)$.

It was proven in [6] that the algebra $T(W)$ is Koszul when $Z_{1}, Z_{2}, \ldots, Z_{d}$ are transversal. If Conjecture 4.2 in that paper is true, then $T(W)$ is Koszul for arbitrary subspaces $Z_{1}, \ldots, Z_{d}$. Such a Koszul duality would lead to new interesting interpretations of the coefficients of $\widetilde{\mathcal{H}}$.

\section{The polarized Schur functor}

6.1 The space $S_{\lambda}\left(Z_{1}, \ldots, Z_{d}\right)$

Assume again that $\mathbf{X}=(X, \mathrm{rk})$ is a polymatroid, $K$ is a field of characteristic 0 , and that we have a realization given by a vector space $V$ and subspaces $V_{x}, x \in X$. Define 
$Z=V^{\star}$, and for every $x \in X$, let $Z_{x}=V_{x}^{\perp}$ be the set of all linear functionals on $V$ vanishing on $V_{x}$. Also, for any $A \subseteq X$, let

$$
Z_{A}=V_{A}^{\perp}=\sum_{x \in A} Z_{x} .
$$

We have

$$
\operatorname{rk}(A)=\operatorname{dim} V-\operatorname{dim} V_{A}=\operatorname{dim} Z_{A}
$$

for all $A \subseteq X$.

Let $\Sigma_{d}$ be the symmetric group on $d$ letters. Its irreducible representations are $T_{\lambda}$ where $\lambda$ runs over all partitions of $d$.

Schur-Weyl duality gives a decomposition

$$
Z^{\otimes d}:=\underbrace{Z \otimes Z \otimes \cdots \otimes Z}_{d} \cong \bigoplus_{\lambda} S_{\lambda} Z \otimes T_{\lambda}
$$

as a representation of $G L(Z) \times \Sigma_{d}$. Let

$$
\pi_{\lambda}: Z^{\otimes d} \rightarrow S_{\lambda} Z \otimes T_{\lambda}
$$

be the $G L(Z) \times \Sigma_{d}$-equivariant projection. There is a unique $\operatorname{GL}(Z) \times \Sigma_{d^{-}}$ equivariant linear map

$$
\theta_{\lambda}: Z^{\otimes d} \otimes T_{\lambda}^{\star} \rightarrow S_{\lambda}(Z)
$$

such that

$$
\theta_{\lambda}(z \otimes \varphi)=(\mathrm{id} \otimes \varphi) \pi_{\lambda}(z)
$$

for every $z \in Z^{\otimes n}$ and $\varphi \in T_{\lambda}^{\star}$. Note that $T_{\lambda}^{\star} \cong T_{\lambda}$ as representations of $\Sigma_{d}$.

Definition 6.1 We define

$$
S_{\lambda}\left(Z_{1}, Z_{2}, \ldots, Z_{d}\right)=\theta_{\lambda}\left(Z_{1} \otimes Z_{2} \otimes \cdots \otimes Z_{d} \otimes T_{\lambda}\right) .
$$

Remark 6.2 For a permutation $\tau \in \Sigma_{d}$ we have

$$
\begin{aligned}
S_{\lambda} & \left(Z_{1}, \ldots, Z_{d}\right) \\
& \left.=\theta_{\lambda}\left(Z_{1} \otimes \cdots \otimes Z_{d}\right) \otimes T_{\lambda}\right) \\
& =\theta_{\lambda}\left(\tau^{-1}\left(Z_{1} \otimes \cdots \otimes Z_{d} \otimes T_{\lambda}\right)\right) \\
& =\theta_{\lambda}\left(\tau^{-1}\left(Z_{1} \otimes \cdots \otimes Z_{d}\right) \otimes T_{\lambda}\right) \\
& \left.=\theta_{\lambda}\left(Z_{\tau(1)} \otimes \cdots \otimes Z_{\tau(d)}\right) \otimes T_{\lambda}\right) \\
& =S_{\lambda}\left(Z_{\sigma(1)}, \ldots, Z_{\sigma(d)}\right) .
\end{aligned}
$$

In other words, $S_{\lambda}\left(Z_{1}, \ldots, Z_{d}\right)$ does not depend on the order of $Z_{1}, \ldots, Z_{d}$. 
Note that

$$
S_{\lambda}(\underbrace{Z, Z, \ldots, Z}_{d})=S_{\lambda}(Z) .
$$

6.2 The connection between $S_{\lambda}\left(Z_{1}, \ldots, Z_{d}\right)$ and $\mathcal{H}[\mathbf{X}](q, t)$

Proposition 6.3 Let us write

$$
\sigma^{n} \mathcal{H}[\mathbf{X}]\left(\sigma^{-1},-1\right)=\sum_{\lambda} a_{\lambda} s_{\lambda}
$$

where $\lambda$ runs over all partitions with $|\lambda| \geq d$. Then we have

$$
a_{\lambda}=\operatorname{dim} S_{\lambda}(Z_{1}, Z_{2}, \ldots, Z_{d}, \underbrace{Z, \ldots, Z}_{|\lambda|-d}) \text {. }
$$

Proof Let $r=|\lambda|$ and $\mathfrak{m}(W)$ be the maximal homogeneous ideal of $R(W)$. The degree $r$ part of $J(W)$ is

$$
J_{1}(W) J_{2}(W) \cdots J_{d}(W) \mathfrak{m}(W)^{r-d} .
$$

Set $U=W \otimes V^{\star}=W \otimes Z$ and $U_{i}=W \otimes Z_{i}$. Then Cauchy's formula tells us that

$$
R(W)=S(W \otimes Z)=\bigoplus_{\lambda} S_{\lambda} W \otimes S_{\lambda} Z
$$

The degree $r$ part of $J(W)$ is

$$
U_{1} \cdot U_{2} \cdots U_{d} \cdot U^{r-d} \subset S_{r}(U)=\bigoplus_{|\lambda|=r} S_{\lambda} W \otimes S_{\lambda} Z .
$$

So if

$$
\pi_{r}^{U}: \underbrace{U \otimes U \otimes \cdots \otimes U}_{r} \rightarrow S_{r}(U)
$$

is the canonical projection, then the degree $r$ part of $J(W)$ is

$$
\pi_{r}(U_{1}, U_{2}, \ldots, U_{d}, \underbrace{U, \ldots, U}_{r-d}) .
$$

Let $\gamma_{\lambda}: S_{r}(U) \rightarrow S_{\lambda} W \otimes S_{\lambda} Z$ be the projection. The isotypic component of $J(W)$ for the representation $S_{\lambda}(W)$ is

$$
\gamma_{\lambda}\left(\pi_{r}\left(U_{1} \otimes \cdots \otimes U_{d} \otimes U^{r-d}\right)\right) .
$$

We have

$$
U^{\otimes r}=(Z \otimes W)^{\otimes r}=Z^{\otimes r} \otimes W^{\otimes r}=\bigoplus_{\lambda} S_{\lambda}(W) \otimes T_{\lambda} \otimes Z^{\otimes r}
$$




$$
\cong \bigoplus_{\lambda} S_{\lambda}(W) \otimes Z^{\otimes r} \otimes T_{\lambda}
$$

If we first project $U^{\otimes r}$ onto $S_{\lambda}(W) \otimes Z^{\otimes r} \otimes T_{\lambda}$ and then we apply

$$
\operatorname{id} \otimes \pi_{\lambda}^{Z}: S_{\lambda}(W) \otimes Z^{\otimes r} \otimes T_{\lambda} \rightarrow S_{\lambda} W \otimes S_{\lambda} Z
$$

then we get a nonzero $\mathrm{GL}(V) \times \mathrm{GL}(Z) \times \Sigma_{r}$ equivariant linear map

$$
U^{\otimes r} \rightarrow S_{\lambda} W \otimes S_{\lambda} Z
$$

This map must be, up to a non-zero scalar, equal to the composition $\gamma_{\lambda} \circ \pi_{r}$. It follows that

$$
\begin{aligned}
\gamma_{\lambda}\left(\pi_{r}\left(U_{1} \otimes \cdots \otimes U_{d} \otimes U^{r-d}\right)\right) & =\mathrm{id} \otimes \pi_{\lambda}\left(S_{\lambda}(W) \otimes Z_{1} \otimes \cdots \otimes Z_{d} \otimes Z^{r-d} \otimes T_{\lambda}\right) \\
& =S_{\lambda} W \otimes S_{\lambda}(Z_{1}, \ldots, Z_{d}, \underbrace{Z, \ldots, Z}_{r-d}) .
\end{aligned}
$$

So, as GL(W)-modules, we have an isomorphism

$$
J(W) \cong \bigoplus_{\lambda} S_{\lambda}(Z_{1}, Z_{2}, \ldots, Z_{d}, \underbrace{Z, \ldots, Z}_{|\lambda|-d}) \otimes S_{\lambda}(W) .
$$

Since $a_{\lambda}$ is the multiplicity of $S_{\lambda} W$ in $J(W)$, we get

$$
a_{\lambda}=\operatorname{dim} S_{\lambda}(Z_{1}, \ldots, Z_{d}, \underbrace{Z, \ldots, Z}_{r-d}) .
$$

For $A \subseteq X$, let us define

$$
S_{\lambda, A}:=S_{\lambda}(V_{x_{1}}, \ldots, V_{x_{k}}, \underbrace{V, \ldots, V}_{|\lambda|-k}),
$$

where $k=|A|$ and $A=\left\{x_{1}, \ldots, x_{k}\right\}$. If $|\lambda|<k$, then we define $S_{\lambda, A}=0$. Define

$$
C_{\lambda, k}=\bigoplus_{|A|=k} S_{\lambda, A}
$$

Then we get

$$
C_{k}=\bigoplus_{\lambda} C_{\lambda, k} \otimes S_{\lambda}(W)
$$

The maps in the complex (26) are GL( $W$ )-equivariant, and by taking the isotypic component for $S_{\lambda(W)}$ we get a complex

$$
0 \rightarrow C_{\lambda, \ell} \otimes S_{\lambda}(W) \rightarrow \cdots \rightarrow C_{\lambda, 1} \otimes S_{\lambda}(W) \rightarrow C_{\lambda, 0} \otimes S_{\lambda}(W) \rightarrow 0
$$


where $\ell=\min \{d,|\lambda|\}$. Since all maps in this complex are $\operatorname{GL}(W)$-equivariant, the complex is obtained from a complex

$$
0 \rightarrow C_{\lambda, \ell} \rightarrow \cdots \rightarrow C_{\lambda, 1} \rightarrow C_{\lambda, 0} \rightarrow 0
$$

by tensoring it by $S_{\lambda}(W)$. The map $\partial_{k}: C_{\lambda, k} \rightarrow C_{\lambda, k-1}$ can be written as $\partial_{k}=$ $\sum_{A, B} \partial_{k}^{A, B}$, where

$$
\partial_{k}^{A, B}: S_{\lambda, A} \rightarrow S_{\lambda, B}
$$

Suppose that $A=\left\{i_{1}, i_{2}, \ldots, i_{k}\right\}$ with $i_{1}<i_{2}<\cdots<i_{k}$, then we have

$$
\partial_{k}^{A, B}:= \begin{cases}0 & \text { if } B \nsubseteq A ; \\ (-1)^{r} \text { id } & \text { if } B=\left\{i_{1}, \ldots, i_{r-1}, i_{r+1}, \ldots, i_{k}\right\} .\end{cases}
$$

Let $H_{\lambda, i}$ be the $i$-th homology group of (34). From

$$
H_{i}(W)=\bigoplus_{\lambda} H_{\lambda, i} \otimes S_{\lambda}(W)
$$

and Corollary 5.3 now follows the following statement.

Corollary 6.4 Suppose that $V_{X}=0$, which means that $Z_{X}=Z$. Write

$$
\mathcal{P}[\mathbf{X}]=\sum_{\lambda}(-1)^{|\lambda|} b_{\lambda} s_{\lambda}
$$

Then we have

$$
\operatorname{dim} H_{\lambda, i}= \begin{cases}0 & \text { if }|\lambda| \neq i \\ b_{\lambda} & \text { if }|\lambda|=i\end{cases}
$$

The dimension of

$$
S_{\lambda} Z=S_{\lambda}(\underbrace{Z, Z, \ldots, Z}_{d})
$$

(where $d=|\lambda|$ ) is exactly the number of Young Tableau of shape $\lambda$ and entries in the set $\{1,2, \ldots, n\}$. In fact, given a basis of $Z$, an explicit basis of $S_{\lambda} Z$ can be given in terms of these Young tableaux (see [14, §8.1, Theorem 1]).

Problem 6.5 Give a combinatorial interpretation of

$$
\operatorname{dim} S_{\lambda}\left(Z_{1}, Z_{2}, \ldots, Z_{d}\right),
$$

perhaps in terms of certain fillings of Young diagrams. Moreover, can one give an explicit basis of $S_{\lambda}\left(Z_{1}, \ldots, Z_{d}\right)$ ?

Such a combinatorial setup might still have a meaning for non-realizable polymatroids. An explicit bases of $S_{d}\left(Z_{1}, \ldots, Z_{d}\right)$ was given in [6, Corollary 5.10] in case the subspaces $Z_{1}, \ldots, Z_{d}$ of $Z$ are generic.

Also, one can ask the same questions for $H_{\lambda}:=H_{\lambda,|\lambda|}$. Such results might prove Conjecture 5.7 . 


\section{Quasi-symmetric functions associated to polymatroids}

\subsection{The Hopf algebras Mat and PolyMat}

Although most of the Hopf algebras in this section can be defined over the integers $\mathbb{Z}$, we will choose to define them over $\mathbb{Q}$ for simplicity. In [32] the matroid Hopf algebra Mat was introduced (see also [9-11]). This construction easily generalizes to polymatroids.

Let us first introduce the Hopf algebra of polymatroids, PolyMat. For a polymatroid $\mathbf{X}=(X, \mathrm{rk})$, we denote its isomorphism class by $[\mathbf{X}]$. As a $\mathbb{Q}$-vector space, PolyMat has a basis consisting of all isomorphism classes of polymatroids. We define a product by

$$
[\mathbf{X}] \cdot[\mathbf{Y}]:=[\mathbf{X} \oplus \mathbf{Y}] .
$$

Also, a coproduct $\Delta:$ PolyMat $\rightarrow$ PolyMat $\otimes_{\mathbb{Q}}$ PolyMat is defined by

$$
\Delta[\mathbf{X}]=\sum_{A \subseteq X}\left[\left.\mathbf{X}\right|_{A}\right] \otimes[\mathbf{X} / A] .
$$

This coproduct is coassociative, but in general not cocommutative. The unit is [Ø] where $\varnothing$ denotes the empty polymatroid. A counit $\epsilon:$ PolyMat $\rightarrow \mathbb{Q}$ is given by

$$
\epsilon([\mathbf{X}])= \begin{cases}1 & \text { if } \mathbf{X}=\emptyset \\ 0 & \text { otherwise. }\end{cases}
$$

The bialgebra PolyMat has a grading such that $[\mathbf{X}]$ has degree $|X|$ for every polymatroid $\mathbf{X}=(X, \mathrm{rk})$. This makes PolyMat into a connected graded bialgebra. It was shown in [27] that one can define an antipode such that PolyMat becomes a Hopf algebra.

Let Mat be the subspace spanned by all [X] where $\mathbf{X}$ is a matroid. Then Mat is sub-Hopf algebra of PolyMat.

\subsection{The Hopf algebra NSym}

Let $N \operatorname{Sym} \mathbb{Q}\left\langle p_{1}, p_{2}, p_{3}, \ldots\right\rangle$ be the ring of noncommutative polynomials in the indeterminates $p_{1}, p_{2}, p_{3}, \ldots$ We define a Hopf algebra structure on NSym as follows. The comultiplication $\Delta: N S y m \rightarrow N S y m \otimes N S y m$ by

$$
\Delta\left(p_{i}\right)=p_{i} \otimes 1+1 \otimes p_{i}
$$

for all $i$. The counit $\epsilon: N S y m \rightarrow \mathbb{Q}$ is defined by

$$
\epsilon\left(p_{i}\right)=0
$$

for all $i$. The antipode is defined by

$$
p_{i} \mapsto-p_{i}
$$


for all $i$. A basis of NSym is given by all noncommutative monomials in $p_{1}, p_{2}, \ldots$ It is also convenient to have a different basis. We define $h_{1}, h_{2}, \ldots$ by the following equality of generating functions in $N \operatorname{Sym}[[t]]$. Define

$$
H(t)=h_{1} t+h_{2} t^{2}+h_{3} t^{3}+\cdots
$$

and

$$
P(t)=p_{1} t+p_{2} t^{2}+p_{3} t^{3}+\cdots .
$$

Then $h_{1}, h_{2}, h_{3}, \ldots$ are defined by

$$
1+H(t)=\exp (P(t))
$$

Here $\exp (t)$ denotes the power series of the exponential function

$$
\exp (t)=1+t+\frac{t^{2}}{2 !}+\frac{t^{3}}{3 !}+\cdots
$$

So we have

$$
h_{k}=\sum_{r=1}^{k} \frac{1}{r !}\left(\sum_{\substack{i_{1}, \ldots, i_{r} \\ i_{1}+\cdots+i_{r}=k}} p_{i_{1}} p_{i_{2}} \cdots p_{i_{r}}\right) .
$$

If $\alpha=\left(i_{1}, \ldots, i_{r}\right)$ is a sequence of positive integers, then we will write $p_{\alpha}$ instead of $p_{i_{1}} p_{i_{2}} \cdots p_{i_{r}}$ and $h_{\alpha}$ instead of $h_{i_{1}} h_{i_{2}} \cdots h_{i_{r}}$. The length of $\alpha$ is $\ell(\alpha):=r$, and we define $|\alpha|=i_{1}+i_{2}+\cdots+i_{r}$. We can rewrite (35) as

$$
h_{k}=\sum_{\substack{\alpha \\|\alpha|=k}} \frac{p_{\alpha}}{\ell(\alpha) !}
$$

Inverting gives

$$
P(t)=\log (1+H(t)),
$$

where

$$
\log (1+t)=t-\frac{t^{2}}{2}+\frac{t^{3}}{3}-\cdots
$$

so

$$
p_{k}=\sum_{r=1}^{k} \frac{(-1)^{r-1}}{r} \sum_{\substack{i_{1}, \ldots, i_{r} \\ i_{1}+\cdots+i_{r}=k}} h_{i_{1}} h_{i_{2}} \cdots h_{i_{r}}
$$

Again, we can rewrite this as

$$
p_{k}=\sum_{\alpha} \frac{(-1)^{\ell(\alpha)-1} h_{\alpha}}{\ell(\alpha)} .
$$


From

$$
\Delta(P(t))=P(t) \otimes 1+1 \otimes P(t)
$$

it follows that

$$
\begin{aligned}
\Delta(1+H(t)) & =\Delta(\exp (P(t))=\Delta(\exp (P(t) \otimes 1+1 \otimes P(t))) \\
& =\exp (P(t) \otimes 1) \exp (1 \otimes P(t))=((1+H(t)) \otimes 1) \cdot(1 \otimes(1+H(t))) \\
& =(1+H(t)) \otimes(1+H(t))
\end{aligned}
$$

inside the ring

$$
N \operatorname{Sym} \otimes N \operatorname{Sym}[[t]]=N \operatorname{Sym}[[t]] \otimes \mathbb{Q}[[t]] \operatorname{NSym}[[t]] .
$$

If we use the convention $h_{0}=1$, then we have

$$
\Delta\left(h_{k}\right)=\sum_{i=0}^{k} h_{i} \otimes h_{k-i} .
$$

The Hopf algebra NSym is not commutative, but it is cocommutative.

7.3 The Hopf algebra QSym

Let $Q S y m$ be the Hopf algebra of quasi-symmetric functions (see $[15,16,18,19]$ ). For a sequence $\alpha=\left(\alpha_{1}, \ldots, \alpha_{r}\right)$ of positive integers we define an element $M_{\alpha} \in$ $\mathbb{Q}\left[x_{1}, x_{2}, \ldots\right]$ by

$$
M_{\alpha}:=\sum_{0<i_{1}<i_{2}<\cdots<i_{r}} x_{1}^{\alpha_{1}} x_{2}^{\alpha_{2}} \cdots x_{r}^{\alpha_{r}} .
$$

The ring $Q S y m$ is the subring of $\mathbb{Q}\left[x_{1}, x_{2}, x_{3}, \ldots\right]$ spanned by all $M_{\alpha}$. The $\mathbb{Q}$-vector space $Q S y m$ is closed under multiplication. We will view $Q S y m$ as the graded dual vector space of NSym where the $\left\{M_{\alpha}\right\}$ form a dual basis of the $\left\{h_{\alpha}\right\}$. As such, QSym is a Hopf algebra in a natural way. Also, let $\left\{P_{\alpha}\right\}$ be a dual basis of $\left\{p_{\alpha}\right\}$. We have that

$$
P_{\alpha} P_{\beta}=\sum_{\gamma} P_{\gamma}
$$

where $\gamma$ runs over all

$$
\left(\begin{array}{c}
\ell(\alpha)+\ell(\beta) \\
\ell(\alpha)
\end{array}\right)
$$

shuffles of $\alpha$ and $\beta$. If $\alpha=\left(\alpha_{1}, \ldots, \alpha_{r}\right)$, then

$$
\Delta\left(P_{\alpha}\right)=\sum_{\beta, \gamma ; \beta \gamma=\alpha} P_{\beta} \otimes P_{\gamma} .
$$

The antipode on QSym is given by

$$
P_{\alpha} \mapsto(-1)^{\ell(\alpha)} P_{\alpha} .
$$


From (36) it follows that

$$
h_{\alpha}=h_{i_{1}} \cdots h_{i_{r}}=\sum_{\substack{\beta_{1}, \ldots, \beta_{r} \\\left|\beta_{1}\right|=i_{1}, \ldots,\left|\beta_{r}\right|=i_{r}}} \frac{p_{\beta_{1} \beta_{2} \cdots \beta_{r}}}{\ell\left(\beta_{1}\right) ! \cdots \ell\left(\beta_{r}\right) !},
$$

where $\alpha=\left(i_{1}, \ldots, i_{r}\right)$. Dualizing (38) gives

$$
P_{\beta}=\sum_{r} \sum_{\substack{\beta_{1} \cdots \beta_{r} \\ \beta=\beta_{1} \cdots \beta_{r}}} \frac{M_{\left|\beta_{1}\right|, \ldots,\left|\beta_{r}\right|}}{\ell\left(\beta_{1}\right) ! \ell\left(\beta_{2}\right) ! \cdots \ell\left(\beta_{r}\right) !} .
$$

From (37) it follows that

$$
p_{\alpha}=p_{i_{1}} \cdots p_{i_{r}}=\sum_{\substack{\beta_{1}, \ldots, \beta_{r} \\\left|\beta_{1}\right|=i_{1}, \ldots,\left|\beta_{r}\right|=i_{r}}} \frac{(-1)^{\ell\left(\beta_{1}\right)+\cdots+\ell\left(\beta_{r}\right)-r} h_{\beta_{1} \beta_{2} \cdots \beta_{r}}}{\ell\left(\beta_{1}\right) \cdots \ell\left(\beta_{r}\right)} .
$$

Dualizing (39) yields

$$
M_{\beta}=\sum_{r} \sum_{\substack{\beta_{1} \cdots \beta_{r} \\ \beta=\beta_{1} \cdots \beta_{r}}}(-1)^{\ell(\beta)-r} \frac{P_{\left|\beta_{1}\right|, \ldots,\left|\beta_{r}\right|}}{\ell\left(\beta_{1}\right) \cdots \ell\left(\beta_{r}\right)}
$$

7.4 Combinatorial Hopf algebras and the invariant $\mathcal{F}[\mathbf{X}]$

Billera, Jia and Reiner defined a homomorphism of Hopf algebras

$$
\mathcal{F}: M a t \rightarrow Q S y m
$$

(see $[3,26]$ ). One way to define this map is using a universal property of QSym.

A combinatorial Hopf algebra (over $\mathbb{Q}$ ) is a pair $(\mathcal{H}, \zeta)$ where $\mathcal{H}=\bigoplus_{d \geq 0} \mathcal{H}_{d}$ is a graded Hopf algebra with $\mathcal{H}_{0}=\mathbb{Q}$ and $\mathcal{H}_{d}$ is finite dimensional for all $\bar{d}$, and $\zeta: \mathcal{H} \rightarrow \mathbb{Q}$ is a character (i.e., an algebra homorphism). A morphism $\varphi:\left(\mathcal{H}^{\prime}, \zeta^{\prime}\right) \rightarrow$ $(\mathcal{H}, \zeta)$ is a Hopf-algebra morphism $\varphi: \mathcal{H}^{\prime} \rightarrow \mathcal{H}$ such that $\zeta \circ \varphi=\zeta^{\prime}$.

Aguiar, Bergeron and Sottile proved that there exists a terminal object in the category of combinatorial Hopf algebras over $\mathbb{Q}$, namely $(Q S y m, \zeta)$ where $\zeta=\zeta_{Q S y m}$ is defined by

$$
\zeta\left(M_{\alpha}\right)= \begin{cases}1 & \text { if } \ell(\alpha) \leq 1 \\ 0 & \text { otherwise }\end{cases}
$$

We can define a character $\zeta=\zeta_{\text {Mat }}$ on Mat by

$$
\zeta([\mathbf{X}])= \begin{cases}1 & \text { if } \mathbf{X} \text { completely splits into loop and coloop matroids; } \\ 0 & \text { otherwise }\end{cases}
$$

Since $\left(Q S y m, \zeta_{Q S y m}\right)$ is terminal, there is a unique homomorphism

$$
\mathcal{F}:\left(M a t, \zeta_{M}\right) \rightarrow\left(Q S y m, \zeta_{Q S y m}\right)
$$


of combinatorial Hopf algebras.

Although $\mathcal{F}$ is a powerful invariant for matroids, it cannot distinguish between a loop and an isthmus.

7.5 The new quasi-symmetric function invariant $\mathcal{G}[\mathbf{X}]$

It sometimes is convention to shift the indices by 1 , so for a vector $a=\left(a_{1}, a_{2}, \ldots, a_{d}\right)$ of nonnegative integers, we define

$$
U_{\left(a_{1}, a_{2}, \ldots, a_{d}\right)}:=P_{a_{1}+1, a_{2}+1, \ldots, a_{d}+1} .
$$

Definition 7.1 We define a $\mathbb{Q}$-linear map

$$
\mathcal{G}: \text { PolyMat } \rightarrow \text { QSym }
$$

defined by

$$
\mathcal{G}[\mathbf{X}]=\sum_{\underline{X}} U_{r(\underline{X})}
$$

where $\underline{X}$ runs over all maximal chains

$$
\underline{X}: \emptyset=X_{0} \subset X_{1} \subset \cdots \subset X_{d}=X
$$

and

$$
r(\underline{X}):=\left(\operatorname{rk}\left(X_{1}\right)-\operatorname{rk}\left(X_{0}\right), \operatorname{rk}\left(X_{2}\right)-\operatorname{rk}\left(X_{1}\right), \ldots, \operatorname{rk}\left(X_{d}\right)-\operatorname{rk}\left(X_{d-1}\right)\right) .
$$

We call $r(\underline{X})$ the rank sequence for $\underline{X}$. The multiset of all $r(\underline{X})$ where $\underline{X}$ runs over all maximal chains in $X$, we will call the rank sequences for $\overline{\mathbf{X}}$. If $\mathbf{X}=(X, \mathrm{rk})$ then there are exactly $|X|$ ! rank sequences.

Lemma 7.2 The linear map $\mathcal{G}$ is a homomorphism of Hopf algebras.

Proof If $\mathbf{X}$ has a rank sequence $\gamma=r(\underline{X})$ and $\gamma=\alpha \beta$, then $\alpha$ is a rank sequence for $\left.\mathbf{X}\right|_{A}$ and $\beta$ is a rank sequence for $\mathbf{X} / A$, where $A=X_{i}$ and $i=\ell(\alpha)$ is the length of $\alpha$. So we have

$$
\begin{aligned}
\mathcal{G} \otimes \mathcal{G} \circ \Delta([\mathbf{X}]) & =\sum_{A \subseteq X} \mathcal{G}\left[\left.\mathbf{X}\right|_{A}\right] \otimes \mathcal{G}[\mathbf{X} / A] \\
& =\sum_{A \subseteq X} \sum_{\alpha} \sum_{\beta} U_{\alpha} \otimes U_{\beta}=\Delta\left(\sum_{\gamma} U_{\gamma}\right)=\Delta(\mathcal{G}[\mathbf{X}]),
\end{aligned}
$$

where $\alpha$ runs over all rank sequences for $\left.\mathbf{X}\right|_{A}, \beta$ runs over all rank sequences of $\mathbf{X} / A$ and $\gamma$ runs over all rank sequences for $\mathbf{X}$.

To see that $\mathcal{G}$ commutes with the product, note that the rank sequences for $\mathbf{X} \oplus \mathbf{Y}$ are exactly all shuffles of rank sequences for $\mathbf{X}$ and $\mathbf{Y}$.

It is easy to verify that $\mathcal{G}$ is compatible with the unit and counit. 
For a vector $\alpha=\left(\alpha_{1}, \alpha_{2}, \ldots, \alpha_{d}\right)$, define

$$
\alpha^{\vee}=\left(1-\alpha_{d}, 1-\alpha_{d-1}, \ldots, 1-\alpha_{1}\right) .
$$

Lemma 7.3 For a matroid $\mathbf{X}=(X$, rk $)$ we have

$$
\mathcal{G}\left[\mathbf{X}^{\vee}\right]=\sum_{\underline{X}} U_{r}(\underline{X})^{\vee}
$$

Proof For a maximal chain $\underline{X}$, define a chain $\underline{X}^{\vee}$ by $X_{i}^{\vee}:=X \backslash X_{d-i}$. Note that

$$
\mathrm{rk}^{\vee}\left(X_{i}\right)=|X|-\operatorname{rk}(X)+\operatorname{rk}\left(X_{d-i}\right)
$$

and

$$
\mathrm{rk}^{\vee}\left(X_{i}^{\vee}\right)-\mathrm{rk}^{\vee}\left(X_{i-1}^{\vee}\right)=1-\left(\operatorname{rk}\left(X_{d-i+1}\right)-\operatorname{rk}\left(X_{d-i}\right)\right) .
$$

If $\alpha$ runs over all rank sequence for $\mathbf{X}$, then $\alpha^{\vee}$ runs over all rank sequences for $\mathbf{X}^{\vee}$.

\section{6 $\mathcal{G}$ specializes to $\mathcal{F}$}

Let us define another character $\gamma: Q S y m \rightarrow \mathbb{Q}$ by

$$
\gamma\left(P_{\alpha}\right)=0
$$

if $\alpha$ is not weakly increasing. Otherwise, write $\alpha=\left(\alpha_{1}^{k_{1}}, \alpha_{2}^{k_{2}}, \cdots, \alpha_{s}^{k_{s}}\right)$ with

$$
\alpha_{1}<\alpha_{2}<\cdots<\alpha_{s},
$$

and define

$$
\gamma\left(P_{\alpha}\right)=\frac{1}{k_{1} ! k_{2} ! \cdots k_{S} !} .
$$

Suppose that $\alpha^{\prime}=\left(\alpha_{1}^{l_{1}}, \cdots, \alpha_{s}^{l_{s}}\right)$. Then

$$
P_{\alpha} P_{\alpha^{\prime}}=\left(\begin{array}{c}
l_{1}+k_{1} \\
k_{1}
\end{array}\right)\left(\begin{array}{c}
l_{2}+k_{2} \\
k_{2}
\end{array}\right) \cdots\left(\begin{array}{c}
l_{s}+k_{s} \\
k_{s}
\end{array}\right) P_{\delta}+P^{\prime},
$$

where $\delta=\left(\alpha_{1}^{k_{1}+l_{1}}, \ldots, \alpha_{s}^{k_{s}+l_{s}}\right)$ and $P^{\prime}$ is a linear combination of $P_{\delta}$ 's where $\delta$ is not weakly increasing. The binomials appear from the fact there are $\left(\begin{array}{c}l_{i}+k_{i} \\ k_{i}\end{array}\right)$ ways to shuffle $\alpha_{i}^{k_{i}}$ and $\alpha_{i}^{l_{i}}$. If we apply $\gamma$ we get

$\gamma\left(P_{\alpha} P_{\alpha^{\prime}}\right)=\gamma\left(P_{\delta}\right)=\frac{\left(\begin{array}{c}l_{1}+k_{1} \\ k_{1}\end{array}\right) \cdots\left(\begin{array}{c}l_{s}+k_{s} \\ k_{s}\end{array}\right)}{\left(l_{1}+k_{1}\right) ! \cdots\left(l_{s}+k_{s}\right) !}=\frac{1}{k_{1} ! \cdots k_{s} !} \cdot \frac{1}{l_{1} ! \cdots l_{s} !}=\gamma\left(P_{\alpha}\right) \gamma\left(P_{\alpha^{\prime}}\right)$.

This shows that $\gamma$ is multiplicative. Since $(Q S y m, \zeta)$ is the terminal object for the combinatorial Hopf algebras, there is a unique morphism of combinatorial Hopf algebras

$$
\theta:(Q S y m, \gamma) \rightarrow(Q S y m, \zeta)
$$


Theorem 7.4 We have

$$
\left.\theta \circ \mathcal{G}\right|_{\text {Mat }}=\mathcal{F},
$$

where $\left.\mathcal{G}\right|_{\text {Mat }}$ is the restriction of $\mathcal{G}$ to Mat.

Proof We claim that

$$
\zeta=\left.\gamma \circ \mathcal{G}\right|_{\text {Mat }}
$$

Suppose that $\mathbf{X}=(X, \mathrm{rk})$ is a matroid with $d:=|X|$ and $n:=\operatorname{rk}(X) \leq d$. Then $\gamma(\mathcal{G}[\mathbf{X}])$ is equal to $\frac{N}{n !(d-n) !}$, where $N$ counts the number of maximal chains

$$
X_{0}=\emptyset \subset X_{1} \subset \cdots \subset X_{d}=X
$$

with

$$
0=\operatorname{rk}\left(X_{0}\right)=\cdots=\operatorname{rk}\left(X_{d-n}\right)=0
$$

and

$$
\operatorname{rk}\left(X_{d-n+i}\right)=i
$$

for $i=1,2, \ldots, n$. Let $Y=X_{d-n}$ and $Z=X \backslash Y$. For a subset $A \subseteq X$, we have

$$
\operatorname{rk}(A) \geq \operatorname{rk}(X)-\operatorname{rk}(X \backslash A)
$$

and

$$
\operatorname{rk}(X \backslash A) \leq \operatorname{rk}(Y \backslash A)+\operatorname{rk}(Z \backslash A)=\operatorname{rk}(Z \backslash A) \leq|Z|-|Z \cap A|=n-|Z \cap A| .
$$

It follows that

$$
\operatorname{rk}(A) \geq \operatorname{rk}(X)-\operatorname{rk}(X \backslash A)=n-(n-|Z \cap A|)=|Z \cap A| .
$$

We also have

$$
\operatorname{rk}(A) \leq \operatorname{rk}(A \cup Y) \leq \operatorname{rk}(Y)+|A \cup Y|-|Y|=|A \cap Z| .
$$

We conclude that

$$
\operatorname{rk}(A)=|A \cap Z|
$$

for all $A \subseteq X$. This implies that

$$
(X, \mathrm{rk})=\underbrace{\mathbf{0} \cdot \mathbf{0} \cdots \mathbf{0}}_{d-n} \cdot \underbrace{\mathbf{1} \cdot \mathbf{1} \cdots \mathbf{1}}_{n},
$$

where $\mathbf{0}$ is the loop matroid, and $\mathbf{1}$ is the isthmus matroid. In particular, if $(X, \mathrm{rk})$ does not split completely, then $\gamma(\mathcal{G}[\mathbf{X}])=0$.

Suppose that $\mathbf{X}=(X, \mathrm{rk})$ splits completely as in (44). Without loss of generality, we may assume that $X=\{1,2, \ldots, d\}$, and $\operatorname{rk}(A)=|A \cap Z|$ where $Y=\{1,2, \ldots, d-$ $n\}$ and $Z=X \backslash Y$. 
A flag

$$
X_{0}=\emptyset \subset X_{1} \subset \cdots \subset X_{d}=X
$$

satisfies (42) and (43) if and only if $X_{d-n}=Y$. There are $(d-n)$ ! flags

$$
\emptyset=X_{0} \subset \cdots \subset X_{d-n}=Y
$$

and $n$ ! flags

$$
Y=X_{d-n} \subset X_{d-n+1} \subset \cdots X_{d}=X .
$$

It follows that $N=n !(d-n) !$, and

$$
\gamma(\mathcal{G}[\mathbf{X}])=\frac{N}{n !(d-n) !}=1 .
$$

It follows that $\left.\gamma \circ \mathcal{G}\right|_{\text {Mat }}=\zeta=\zeta([\mathbf{X}])$. By the uniqueness, we get $\left.\theta \circ \mathcal{G}\right|_{\text {Mat }}=\mathcal{F}$.

Note that

$$
\mathcal{G}(\text { Mat }) \subseteq Q \mathrm{Sym}_{2},
$$

where $\mathrm{QSym}_{2}$ is the sub-Hopf algebra of $Q S y m$ spanned by all $Q_{\alpha}$ 's where $\alpha$ is a sequence of 0's and 1's. The algebra $Q S y m_{2}$ is the graded dual of the Hopf algebra $\mathbb{Q}\left\langle p_{1}, p_{2}\right\rangle$. Now $\theta$ restricts to a homomorphism

$$
\theta_{2}: \mathrm{QSym}_{2} \rightarrow \text { QSym }
$$

Proposition 7.5 The homomorphism $\theta_{2}$ is surjective, and the kernel of $\theta_{2}$ is the principal ideal generated by $P_{(2)}-P_{(1)}=U_{(1)}-U_{(0)}$.

Proof The surjectivity follows from the fact that $\mathcal{F}$ is surjective. We choose the grading on $Q S y m_{2}$ where $P_{\alpha}$ has degree $\ell(\alpha)$. There are $2^{d}$ basis elements $P_{\alpha}$ of degree $d$. So the Hilbert series of the $Q S y m_{2}$ is

$$
1+2 t+2^{2} t^{2}+\cdots=\frac{1}{1-2 t} .
$$

Note that $\mathrm{QSym}_{2}$ is not finitely generated as a commutative algebra.

On $Q S y m$, we choose the grading where $P_{\alpha}$ has degree $|\alpha|$. There is one basis element of degree 0 , namely $P_{()}$and for $d>0$ there are $2^{d-1}$ basis elements of degree $d$, because there are $2^{d-1}$ decompositions of $d$. So the Hilbert series of QSym with this grading is

$$
1+t+2 t^{2}+2^{2} t^{3}+\cdots 1+\frac{t}{1-2 t}=\frac{1-t}{1-2 t} .
$$

Therefore, the Hilbert series of the kernel of $\theta_{2}$ is

$$
\frac{1}{1-2 t}-\frac{1-t}{1-2 t}=\frac{t}{1-2 t} \text {. }
$$


The kernel contains the principal ideal $\left(P_{(2)}-P_{(1)}\right)$. It is not hard to see that $P_{(2)}-$ $P_{(1)}$ is not a zero divisor, so the Hilbert series of the principal ideal is $\frac{t}{1-2 t}$. Since this is equal to the Hilbert series of the kernel of $\theta_{2}$ we must have

$$
\operatorname{ker} \theta_{2}=\left(P_{(2)}-P_{(1)}\right) .
$$

\section{7 $\mathcal{G}$ specializes to $\mathcal{H}$}

Theorem 7.6 There exists a homomorphism $\tau: Q S y m \rightarrow S y m[q, t]$ of commutative algebras such that $\tau(\mathcal{G}[\mathbf{X}])=\mathcal{H}[\mathbf{X}]$ for every polymatroid $\mathbf{X}$.

Proof We will inductively define a symmetric function $\mathcal{P}(\alpha)$ for any vector $\alpha=\left(\alpha_{1}, \ldots, \alpha_{d}\right)$ of nonnegative integers as follows. We define $\mathcal{P}()=1$. Then $\mathcal{P}\left(\alpha_{1}, \ldots, \alpha_{d}\right)$ is the unique symmetric function of degree $<d$ such that

$$
\sum_{i=0}^{d}\left(\begin{array}{l}
d \\
i
\end{array}\right) \mathcal{P}\left(\alpha_{1}, \ldots, \alpha_{i}\right)(-1)^{i} \sigma^{-\alpha_{1}-\cdots-\alpha_{i}}
$$

vanishes in degree $<d$. For a vector $\alpha=\left(\alpha_{1}, \ldots, \alpha_{d}\right)$ and $i<d$, let $\alpha^{[i]}=$ $\left(\alpha_{1}, \ldots, \alpha_{i}\right)$ be the truncated vector. So (45) becomes

$$
\sum_{i=0}^{d}\left(\begin{array}{l}
d \\
i
\end{array}\right) \mathcal{P}\left(\alpha^{[i]}\right)(-1)^{i} \sigma^{-\left|\alpha^{[i]}\right|} .
$$

Define

$$
\widetilde{\mathcal{P}}[\mathbf{X}]=\frac{1}{d !} \sum_{\underline{X}} \mathcal{P}(r(\underline{X}))
$$

for every polymatroid $\mathbf{X}=(X, \mathrm{rk})$ such that $d=|X|$. Here $\underline{X}$ runs over all maximal chains in $X$.

We claim that $\mathcal{P}[\mathbf{X}]=\widetilde{\mathcal{P}}[\mathbf{X}]$. The claim is clearly true when $|X|=0$ or $|X|=1$. Note that $\widetilde{\mathcal{P}}[\mathbf{X}]$ is a symmetric polynomial of degree $<d=|X|$. To prove the claim it suffices to show that

$$
\sum_{A \subseteq X} \widetilde{\mathcal{P}}\left[\left.\mathbf{X}\right|_{A}\right](-1)^{|A|} \sigma^{-\operatorname{rk}(A)}
$$

vanishes in degree $<d$. The symmetric polynomial (47) is equal to

$$
\sum_{i=0}^{d} \sum_{\substack{A \subseteq X \\|A|=i}} \frac{1}{i !} \sum_{\underline{A}} \mathcal{P}(r(\underline{A}))(-1)^{i} \sigma^{-\operatorname{rk}(A)},
$$

where $\underline{A}$ runs over all maximal chains in $A$. Every such chain $\underline{A}$ can be extended to $(d-i)$ ! maximal chains in $X$. Therefore, (49) is equal to

$$
\sum_{i=0}^{d} \frac{1}{i !(d-i) !} \sum_{\underline{X}} \mathcal{P}\left(r(\underline{X})^{[i]}\right)(-1)^{i} \sigma^{-\left|r(\underline{X})^{[i]}\right|}
$$




$$
=\frac{1}{d !} \sum_{\underline{X}} \sum_{i=0}^{d}\left(\begin{array}{l}
d \\
i
\end{array}\right) \mathcal{P}\left(r(\underline{X})^{[i]}\right)(-1)^{i} \sigma^{-\left|r(\underline{X})^{[i]}\right|}
$$

which vanishes in degree $<d$.

For a vector $\alpha=\left(\alpha_{1}, \ldots, \alpha_{d}\right)$, define

$$
\tau\left(U_{\alpha}\right)=\sum_{i=0}^{d} \frac{1}{i !(d-i) !} \mathcal{P}\left(\alpha^{[i]}\right) q^{\left|\alpha^{[i]}\right|} t^{i}
$$

If $\mathbf{X}=(X, \mathrm{rk})$ is a polymatroid with $|X|=d$, then we have

$$
\tau(\mathcal{G}[\mathbf{X}])=\tau\left(\sum_{\underline{X}} U_{r(\underline{X})}\right)=\sum_{\underline{X}} \tau\left(U_{r(\underline{X})}\right)=\sum_{\underline{X}} \sum_{i=0}^{d} \frac{1}{i !(d-i) !} \mathcal{P}\left(r(\underline{X})^{[i]}\right) q^{\left|r(\underline{X})^{[i]}\right|} t^{i} .
$$

For every subset $A \subseteq X$ with $|A|=i$, and every maximal chain $\bar{A}$ in $A$ there are exactly $(d-i)$ ! maximal chains $\bar{X}$ in $X$ extending $\bar{A}$. Therefore, (51) is equal to

$$
\sum_{i=0}^{d} \sum_{A \subseteq X ;|A|=i} \frac{1}{i !} \sum_{\underline{A}} \mathcal{P}(r(\underline{A})) q^{\mathrm{rk}(A)} t^{|A|}=\sum_{A \subseteq X} \mathcal{P}\left[\left.\mathbf{X}\right|_{A}\right] q^{\mathrm{rk}(A)} t^{|A|}=\mathcal{H}[\mathbf{X}](q, t) .
$$

Corollary 7.7 The quasi-symmetric function $\mathcal{F}[\mathbf{X}]$ specializes to $\mathcal{P}[\mathbf{X}]$ for matroids $\mathbf{X}$.

Proof We define $\xi: Q$ Sym $_{2} \rightarrow$ Sym by

$$
\xi\left(Q_{\alpha}\right)=\left.t^{\ell(\alpha)} \tau\left(Q_{\alpha}\right)\left(1, t^{-1}\right)\right|_{t=0} .
$$

One easily verifies that $\xi$ is a homomorphism of algebras, and

$$
\xi(\mathcal{G}[\mathbf{X}])=\left.\mathcal{H}[\mathbf{X}]\left(1, t^{-1}\right) t^{|X|}\right|_{t=0}=\mathcal{P}[\mathbf{X}]
$$

for every matroid $\mathbf{X}=(X, \mathrm{rk})$. Since $Q_{(1)}-Q_{(0)}$ lies in the kernel of $\xi$, $\xi$ factors through $\theta: Q \operatorname{Sym}_{2} \rightarrow Q S y m \cong Q \operatorname{Sym}_{2} /\left(Q_{(1)}-Q_{(0)}\right)$, say $\xi=\eta \circ \theta$. Then we have

$$
\mathcal{P}[\mathbf{X}]=\xi(\mathcal{G}[\mathbf{X}])=\eta(\theta(\mathcal{G}[\mathbf{X})]))=\eta(\mathcal{F}[\mathbf{X}]) .
$$

\subsection{Speyer's invariant}

For a matroid $\mathbf{X}$ David Speyer defined an interesting polynomial $g_{\mathbf{X}}(t)$. It has the multiplicative property $\left(g_{\mathbf{X}_{1} \oplus \mathbf{X}_{\mathbf{2}}}(t)=g_{\mathbf{X}_{\mathbf{1}}}(t) g_{\mathbf{X}_{\mathbf{2}}}(t)\right)$, it is invariant under matroid-duality and has various other nice properties.

Conjecture 7.8 The invariant $\mathcal{G}$ specializes to Speyer's invariant. 


\section{Polymatroid base polytopes}

8.1 The valuative property of $\mathcal{G}$

We will denote $\{1,2, \ldots, n\}$ by $\underline{n}$. For a polymatroid $\mathbf{X}=(\underline{n}$, rk $)$ we define its base polytope $Q(\mathrm{rk})=Q_{X}(\mathrm{rk}) \subset \mathbb{R}^{n}$ by

$$
Q(\mathrm{rk})=\left\{v \in \mathbb{R}^{n} \mid \sum_{i=1}^{n} v_{i}=\operatorname{rk}(\underline{n}) \text { and } \forall A \subseteq \underline{n}, \sum_{i \in A} v_{i} \leq \operatorname{rk}(A)\right\} .
$$

The $i$-th basis vector is denoted by $e_{i}$.

Theorem 8.1 ([20]) A compact convex polytope in $\mathbb{R}^{n}$ is the base polytope of a polymatroid if and only if every vertex of the polytope has nonnegative integer coordinates, and every edge is parallel to $e_{j}-e_{k}$ for some $j \neq k$.

For a compact convex polytope $\Pi \subset \mathbb{R}^{n}$, its characteristic function $[\Pi]: \mathbb{R}^{n} \rightarrow \mathbb{R}$ is defined by

$$
[\Pi](x)= \begin{cases}1 & \text { if } x \in \Pi ; \\ 0 & \text { if } x \notin \Pi .\end{cases}
$$

Let $\mathcal{K}\left(\mathbb{R}^{n}\right)$ be the $\mathbb{R}$-vector space spanned by all [ח] where $\Pi$ is a compact convex polytope. The Euler characteristic is a linear function $\chi: \mathcal{K}\left(\mathbb{R}^{n}\right) \rightarrow \mathbb{R}$ such that $\chi([\Pi])=1$ for every compact convex polytope $\Pi$ (see [2, Theorem 7.4] where $\chi$ is defined for the slightly larger algebra of closed convex sets).

Definition 8.2 Suppose that $V$ is a $\mathbb{Q}$-vector space. A $\mathbb{Q}$-linear map $f:$ PolyMat $\rightarrow$ $V$ is called valuative if it has the following property. For a finite set $X$ and polymatroids $\mathbf{X}=\left(X, \mathrm{rk}_{i}\right), i=1,2, \ldots, r$ and rational numbers $a_{1}, \ldots, a_{r} \in \mathbb{Q}$ such that

$$
\sum_{i=1}^{r} a_{i}\left[Q\left(\mathrm{rk}_{i}\right)\right]=0
$$

we have that

$$
\sum_{i=1}^{r} a_{i} f\left[\mathbf{X}_{i}\right]=0 .
$$

Moreover, let us call $f$ additive if it is valuative and $f([\mathbf{X}])=0$ whenever the polymatroid base polytope $Q(\mathrm{rk})$ of $\mathbf{X}=(X, \mathrm{rk})$ has dimension $<n-1$.

\section{Theorem 8.3}

$$
\mathcal{G}: \text { PolyMat } \rightarrow \text { QSym }
$$

is valuative.

The proof of the theorem is in the next subsection.

Corollary 8.4 Since $\mathcal{G}$ specializes to $\mathcal{H}$ and $\mathcal{P}$, these invariants are valuative as well. 
A polymatroid base decomposition is a decomposition

$$
Q(\mathrm{rk})=\bigcup_{i=1}^{r} Q\left(\mathrm{rk}_{i}\right)
$$

such that

$$
Q\left(\mathrm{rk}_{i}\right) \cap Q\left(\mathrm{rk}_{j}\right)
$$

is a common face of $Q\left(\mathrm{rk}_{i}\right)$ and $Q\left(\mathrm{rk}_{j}\right)$ for $i \neq j$. Let us call such a decomposition proper if $r>1$ and $Q\left(\mathrm{rk}_{i}\right) \nsubseteq Q\left(\mathrm{rk}_{j}\right)$ for all $i \neq j$. The polytope $Q(\mathrm{rk})$ is called indecomposable if it does not have a proper decomposition. For a fixed base field $K$, a polymatroid is called rigid if it has only finitely many realizations over $K$ as a subspace arrangement up to isomorphism. The work of Lafforgue implies that a realizable matroid is rigid if and only if its matroid base polytope is indecomposable (see [24, 25]). It is therefore of interest to know whether a given matroid polytope is indecomposable. Valuative and additive invariants can be useful to determine whether a matroid polytope is decomposable. For a valuative invariant $f$, we have, by the inclusion-exclusion principle

$$
f(\mathrm{rk})=\sum_{k=1}^{r}(-1)^{k-1} \sum_{i_{1}<i_{2}<\cdots<i_{k}} f\left(\mathrm{rk}_{i_{1}, i_{2}, \ldots, i_{k}}\right),
$$

where $\mathrm{rk}_{i_{1}, \ldots, i_{k}}$ is the rank function whose polymatroid polytope is

$$
Q\left(\mathrm{rk}_{i_{1}}\right) \cap \cdots \cap Q\left(\mathrm{rk}_{i_{k}}\right) \text {. }
$$

If $f$ is additive, then we have

$$
f(\mathrm{rk})=\sum_{i=1}^{r} f\left(\mathrm{rk}_{i}\right) .
$$

Additive invariants can also be constructed from the Billera-Jia-Reiner quasisymmetric function (see [3]).

Conjecture 8.5 Is $\mathcal{G}$ universal with respect to the valuative property? I.e., is it true that for every $\mathbb{Q}$-linear valuative map $f:$ PolyMat $\rightarrow V$ there exists a $\mathbb{Q}$-linear map $\psi: Q S y m \rightarrow V$ such that $\psi \circ \mathcal{G}=f$ ?

\subsection{The proof of Theorem 8.3}

The basis vectors of $\mathbb{R}^{n}$ are denoted by $e_{1}, \ldots, e_{n}$. Let $\Delta$ be the $(n-2)$-dimensional simplex spanned by $e_{1}-e_{2}, e_{2}-e_{3}, \ldots, e_{n-1}-e_{n}$.

Lemma 8.6 Choose $\varepsilon$ such that $0<\varepsilon<1$. For $v \in \mathbb{Z}^{n}$, and a rank function rk : $\operatorname{Pow}(X) \rightarrow \mathbb{R}$, the following statements are equivalent.

1. $\sum_{i=1}^{s} v_{i}=\operatorname{rk}(\underline{s})$ for $s=1,2, \ldots, n$; 
2. $v \in Q(\mathrm{rk})$, and $v+\varepsilon\left(e_{j}-e_{k}\right) \notin Q$ (rk) for all $j<k$;

3. $(v+\varepsilon \Delta) \cap Q(\mathrm{rk})=\emptyset$ and $v \in Q$ (rk).

Proof $(1) \Rightarrow(2)$ : Suppose that (1) holds. Suppose that $A=\left\{i_{1}, \ldots, i_{s}\right\}$ with $i_{1}<$ $\cdots<i_{s}$. Then we have

$\operatorname{rk}\left(\left\{i_{1}, \ldots, i_{t}\right\}\right)-\operatorname{rk}\left(\left\{i_{1}, i_{2}, \ldots, i_{t-1}\right\}\right) \leq \operatorname{rk}\left(\left\{1,2, \ldots, i_{t}\right\}\right)-\operatorname{rk}\left(\left\{1,2, \ldots, i_{t}-1\right\}\right)=v_{i_{t}}$

by the submodular property of the rank function.

Summing (53) for $t=1,2, \ldots, s$ gives

$$
\operatorname{rk}\left(\left\{i_{1}, \ldots, i_{s}\right\}\right) \leq v_{i_{1}}+\cdots+v_{i_{s}}=\sum_{i \in A} v_{i} .
$$

This implies that $v \in Q$ (rk). If $j<k$ and $w=v+\varepsilon\left(e_{j}-e_{k}\right)$, then we have

$$
\sum_{i=1}^{j} w_{i}=\sum_{i=1}^{j} v_{i}+\varepsilon=\operatorname{rk}(\underline{j})+\varepsilon>\operatorname{rk}(\underline{j}),
$$

so $w \notin Q$ (rk). This proves that (2) holds.

(2) $\Rightarrow$ (1): Conversely, assume that (2) holds. A subset $S \subseteq \underline{n}$ is called tight if $\sum_{i \in S} v_{i}=\operatorname{rk}(S)$. Clearly, $\underline{n}$ and $\emptyset$ are tight. If $S, T$ are tight, then

$$
\begin{aligned}
\operatorname{rk} & (S \cup T)+\operatorname{rk}(S \cap T) \leq \operatorname{rk}(S)+\operatorname{rk}(T) \\
& =\sum_{i \in S} v_{i}+\sum_{i \in T} v_{i} \\
& =\sum_{i \in S \cap T} v_{i}+\sum_{i \in S \cup T} v_{i} \leq \operatorname{rk}(S \cap T)+\operatorname{rk}(S \cup T),
\end{aligned}
$$

so all inequalities are equalities, and $S \cup T$ and $S \cap T$ are tight as well.

Suppose that $j<k$ and set $w=v+\varepsilon\left(e_{j}-e_{k}\right)$. Because $g \notin Q(\mathrm{rk})$, there exists a set $A_{j, k}$ such that

$$
\sum_{i \in A_{j, k}} w_{i}>\operatorname{rk}\left(A_{j, k}\right)
$$

Since

$$
\sum_{i \in A_{j, k}} v_{i} \leq \operatorname{rk}\left(A_{j, k}\right),
$$

we must have $j \in A_{j, k}$ and $k \notin A_{j, k}$. We obtain

$$
\operatorname{rk}\left(A_{j, k}\right) \geq \sum_{i \in A_{j, k}} v_{i}=\sum_{i \in A_{j, k}} w_{i}-\varepsilon>\operatorname{rk}\left(A_{j, k}\right)-\varepsilon .
$$

Because $v$ is an integer vector, the first inequality is an equality and $A_{j, k}$ is tight. To prove (1) we need to show that $\underline{i}$ is tight for $i=0,1, \ldots, n$. We do this by induction on 
$i$, the case $i=0$ being trivial. Suppose that $i>0$ and $i-1$ is tight. Then $\underline{i-1} \cup A_{i, k}$ is tight for $k=i+1, \ldots, n$. We have

$$
\underline{i}=\bigcap_{k=i+1}^{n}\left(\underline{i-1} \cup A_{i, k}\right)
$$

because $\underline{i} \subseteq i-1 \cup A_{i, k}$ for all $i$, and $k \notin i-1 \cup A_{i, k}$. Hence $\underline{i}$ is tight.

(3) $\Rightarrow$ (2): This implication is clear because $\left(e_{j}-e_{k}\right) \in \Delta$ for all $j<k$.

(2) $\Rightarrow$ (3): Suppose $v \in Q$ (rk) and $v+\varepsilon\left(e_{j}-e_{k}\right) \notin Q$ (rk) for all $j<k$. Suppose that $v+\delta\left(e_{j}-e_{k}\right) \in Q(\mathrm{rk})$ for some $j, k$ with $j<k$ and $\delta>0$. Set $z:=e_{j}-e_{k}$. If the inequality

$$
\sum_{i \in A} v_{i} \leq \mathrm{rk}(A)
$$

is an equality, then

$$
\operatorname{rk}(A)+\delta \sum_{i \in A} z_{i}=\sum_{i \in A}\left(v_{i}+\delta z_{i}\right) \leq \operatorname{rk}(A)
$$

because $v+\delta z \in Q(\mathrm{rk})$. So we obtain

$$
\sum_{i \in A} z_{i} \leq 0
$$

Therefore, we have

$$
\sum_{i \in A}\left(v_{i}+\varepsilon z_{i}\right) \leq \operatorname{rk}(A) .
$$

If (55) is not tight, then

$$
\sum_{i \in A} v_{i} \leq \mathrm{rk}(A)-1
$$

and

$$
\sum_{i \in A}\left(v_{i}+\varepsilon z_{i}\right) \leq \operatorname{rk}(A)-1+\varepsilon \sum_{i \in A} z_{i} \leq \operatorname{rk}(A)-1+\varepsilon \leq \operatorname{rk}(A) .
$$

So we conclude that

$$
\sum_{i \in A}\left(v_{i}+\varepsilon z_{i}\right) \leq \mathrm{rk}(A)
$$

for all subsets $A \subseteq \underline{n}$. So $v+\varepsilon z \in Q$ (rk), but this contradicts our assumptions. We conclude that $v+\delta\left(e_{j}-e_{k}\right) \notin Q$ (rk) for every $j<k$ and every $\delta>0$.

Suppose that $v$ lies in the interior of a face of positive dimension of $Q$ (rk). This face is parallel to $e_{j}-e_{k}$ for some $j<k$. This means that there exists a $\delta>0$ such that $v+\delta\left(e_{j}-e_{k}\right), v-\delta\left(e_{j}-e_{k}\right) \in Q$ (rk) for some $\delta>0$. This gives a contradiction, therefore $v$ must be a vertex of the polytope $Q(\mathrm{rk})$. Let $v_{1}, v_{2}, \ldots, v_{r}$ be other vertices of $Q(\mathrm{rk})$ such that the edges of $Q(\mathrm{rk})$ meeting at $v$ are $v v_{1}, v v_{2}, \ldots, v v_{r}$. For every 
$v_{i}, v-v_{i}$ is a positive multiple of $e_{k}-e_{j}$ for some $j<k$. This means that $Q(\mathrm{rk})$ is contained in cone

$$
C:=v+\mathbb{R}_{\geq 0}\left(e_{2}-e_{1}\right)+\mathbb{R}_{\geq 0}\left(e_{3}-e_{2}\right)+\cdots+\mathbb{R}_{\geq 0}\left(e_{n}-e_{n-1}\right),
$$

where $\mathbb{R}_{\geq 0}$ denotes the nonnegative real numbers. We conclude that

$$
(v+\varepsilon \Delta) \cap Q(\mathrm{rk}) \subseteq(v+\varepsilon \Delta) \cap C=\emptyset .
$$

So (3) follows.

For $v \in \mathbb{Z}^{n}$, define a valuation $\mu_{v}: \mathcal{K}(\mathbb{R}) \rightarrow \mathbb{R}$ by

$$
\mu_{v}(h)=h(v)-\lim _{\varepsilon \downarrow 0} \chi([v+\varepsilon \Delta] \cdot h) .
$$

Let

$$
r=\left(r_{1}, r_{2}, \ldots, r_{n}\right),
$$

where $r_{i}=\operatorname{rk}(\underline{i})-\operatorname{rk}(\underline{i-1})$ for all $i$.

Corollary 8.7 We have

$$
\mu_{v}([\operatorname{Poly}(\mathrm{rk})])= \begin{cases}1 & \text { if } v=r \\ 0 & \text { otherwise. }\end{cases}
$$

Proof Suppose that $v=r$. By Lemma 8.6, we have $v \in Q(\mathrm{rk})$ and $(v+\varepsilon \Delta) \cap$ $Q($ rk $)=\emptyset$. Therefore, we get

$$
\chi([v+\varepsilon \Delta] \cdot[Q(\mathrm{rk})])=\chi([(v+\varepsilon \Delta) \cap Q(\mathrm{rk})])=\chi([\varnothing])=\chi(0)=0
$$

and $[Q(\mathrm{rk})](v)=1$, so $\mu_{v}([Q(\mathrm{rk})])=1$.

Suppose that $v \neq r$. Assume that $v \notin Q$ (rk). Since $Q(\mathrm{rk})$ is closed, there exists a $\delta>0$ such that

$$
(v+(\varepsilon \Delta)) \cap Q(\mathrm{rk})
$$

for all $\varepsilon$ with $0<\varepsilon<\delta$. This implies that $\mu_{v}([Q(\mathrm{rk})])=0$.

Suppose that $v \in Q(\mathrm{rk})$. Then $(v+\varepsilon \Delta) \cap Q(\mathrm{rk})$ is a closed nonempty convex polytope. Hence we have

$$
\chi([v+\varepsilon \Delta] \cdot[Q(\mathrm{rk})])=1 .
$$

Therefore, we conclude that $\mu_{v}([Q(\mathrm{rk})])=1-1=0$.

Proof of Theorem 8.3 The symmetric group $\Sigma_{n}$ acts on $\mathbb{R}^{n}$ by permuting the coordinates. Define

$$
\mu_{v}^{\sigma}(h)=\mu_{v}(h \circ \sigma)
$$


for every $\sigma \in \Sigma_{n}$ and every $h \in \mathcal{K}(\mathbb{R})$. We have that

$$
\begin{aligned}
& \mu_{v}^{\sigma}([Q(\mathrm{rk})]) \\
& \quad=\mu_{v}([Q(\mathrm{rk} \circ \sigma)]) \\
& \quad= \begin{cases}1 & \text { if } v_{i}=\operatorname{rk}(\{\sigma(1), \ldots, \sigma(i)\})-\operatorname{rk}(\{\sigma(1), \ldots, \sigma(i-1)\}) \text { for all } i \\
0 & \text { otherwise }\end{cases}
\end{aligned}
$$

Define

$$
M_{v}=\sum_{\sigma \in \Sigma_{n}} \mu_{v}^{\sigma} .
$$

From the definition of $\mathcal{G}$ follows that

$$
\mathcal{G}[\mathbf{X}]=\sum_{v} M_{v}([Q(\mathrm{rk})]) U_{v} .
$$

From the linearity of $M_{v}$ and $\mathcal{G}$ it follows that

$$
\sum_{i} a_{i} \mathcal{G}\left[\left(\{1, \ldots, n\}, \mathrm{rk}_{i}\right)\right]=0
$$

whenever

$$
\sum_{i} a_{i}\left[Q\left(\mathrm{rk}_{i}\right)\right]=0
$$

This completes the proof of the theorem.

\section{Future directions}

For a polymatroid $\mathbf{X}$ we defined symmetric functions $\mathcal{P}[\mathbf{X}]$ and $\mathcal{H}[\mathbf{X}]$. In the case where the polymatroid comes from a subspace arrangement, we gave interpretations of the coefficients of these symmetric functions in terms of the Hilbert series and the minimal free resolution of the associated product ideal, and in terms of the polarized Schur functor. We hope for similar interpretations and nonnegativity results in the case where the polymatroid is not realizable (Conjecture 5.7). We also defined a quasi-symmetric function $\mathcal{G}[\mathbf{X}]$. This invariant has many interesting properties, and it specializes to $\mathcal{P}[\mathbf{X}], \mathcal{H}[\mathbf{X}]$ and to the Billera-Jia-Reiner quasi-symmetric function $\mathcal{F}[\mathbf{X}]$. We would like to know whether $\mathcal{G}[\mathbf{X}]$ specializes to Speyer's invariant in [34] (Conjecture 7.8). The invariant $\mathcal{G}$ behaves valuatively with respect to (poly-)matroid base polytope decompositions. We wonder whether $\mathcal{G}$ is universal with this property (Conjecture 8.5).

Acknowledgement The author would like to thank Nathan Reading, Frank Sottile, David Speyer for inspiring discussions and helpful suggestions. 


\section{References}

1. Aguiar, M., Bergeron, N., Sottile, F.: Combinatorial Hopf algebras and generalized DehnSommerville relations. Compos. Math. 142(1), 1-30 (2006)

2. Barvinok, A.: A Course in Convexity. Graduate Studies in Mathematics, vol. 54. AMS, Providence (2002)

3. Billera, L.J., Jia, X., Reiner, V.: A quasisymmetric function for matroids, European J. Comb. (2008, to appear). arXiv:math/0606646

4. Brylawski, T.H.: The Tutte-Grothendieck ring. Algebra Universalis 2, 375-388 (1972)

5. Brylawski, T.H.: Intersection Theory for graphs. J. Combin. Theory Ser. 3(2), 233-246 (1981)

6. Conca, A.: Linear spaces, transversal polymatroids and ASL domains. J. Algebraic Combin. 25(1), 25-41 (2007)

7. Conca, A., Herzog, J.: Castelnuovo-Mumford regularity of products of ideals. Collect. Math. 54(2), 137-152 (2003)

8. Crapo, H.: The Tutte polynomial. Aequationes Math. 3, 211-229 (1969)

9. Crapo, H., Schmitt, W.: A free subalgebra of the algebra of matroids, European J. Comb. 26(7) 10661085

10. Crapo, H., Schmitt, W.: The free product of matroids. European J. Comb. 26(7), 1060-1065 (2005)

11. Crapo, H., Schmitt, W.: A unique factorization theorem for matroids. J. Comb. Theory, Series A 112(2), 222-249 (2005)

12. Derksen, H.: Hilbert series of subspace arrangements. J. Pure Appl. Algebra 209(1), 91-98 (2007)

13. Eisenbud, D.: Commutative Algebra with a View toward Algebraic Geometry. Graduate Texts in Mathematics, vol. 150. Springer, Berlin (1995)

14. Fulton, W.: Young Tableaux. London Mathematical Society Student Texts, vol. 35. Cambridge University Press, Cambridge (1997)

15. Gessel, I.: Multipartite $P$-partitions and inner products of skew Schur functions. In: Combinatorics and Algebra, Boulder, CO, 1983. Contemp. Math., vol. 34, pp. 289-317. Amer. Math. Soc., Providence (1984)

16. Gelfand, I., Krob, D., Lascoux, A., Leclerc, B., Retakh, V., Thibon, J.-Y.: Noncommutative symmetric functions. Adv. Math. 112(2), 218-348 (1995)

17. Goresky, M., MacPherson, R.: Stratified Morse Theory. Ergebnisse der Mathematik und ihrer Grenzgebiete (3), vol. 14. Springer, Berlin (1988)

18. Hazewinkel, M.: Symmetric functions, noncommutative symmetric functions, and quasisymmetric functions, Monodromy and differential equations (Moscow, 2001). Acta Appl. Math. 75(1-3), 55-83 (2003)

19. Hazewinkel, M.: Symmetric functions, noncommutative symmetric functions and quasisymmetric functions. II. Acta Appl. Math. 85(1-3), 319-340 (2005)

20. Herzog, J., Hibi, T.: Discrete polymatroids. J. Algebraic Combin. 16, 239-268 (2002)

21. Hilbert, D.: Über die Theorie von algebraischen Formen. Math. Ann. 36, 313-373 (1890)

22. MacDonald, I.G.: Symmetric Functions and Hall Polynomials. Oxford Mathematical Monographs. Clarendon Press, Oxford (1995)

23. Knuth, D.E.: The Art of Computer Programming, vol. 1, 3rd edn. Addison-Wesley, Reading (1997)

24. Lafforgue, L.: Pavages des simplixes, schémas de graphes recollés et compactification des $\mathrm{PGL}_{r}^{n+1} / \mathrm{PGL}_{r}$. Invent. Math. 136(1), 233-271 (1999)

25. Lafforgue, L.: Chirurgie des Grassmanniennes. CRM Monograph Series, vol. 19. American Mathematical Society, Providence (2003)

26. Luoto, K.: A matroid-friendly basis for quasisymmetric functions. J. Comb. Theory Ser. A 115(5), 777-787 (2008). arXiv:0704.0836 [math.CO]

27. Milnor, J.W., Moore, J.C.: On the structure of Hopf algebras. Ann. Math. 81, 211-264 (1965)

28. Noble, S.D., Welch, D.J.A.: A weighted graph polynomial from chromatic invariants of knots. Ann. Inst. Fourier (Grenoble) 49(3), 1057-1087 (1999)

29. Orlik, P., Solomon, L.: Combinatorics and topology of complements of hyperplanes. Invent. Math. 56(2), 167-189 (1980)

30. Oxley, J.G.: Matroid Theory. Oxford University Press, New York (1992)

31. Sarmiento, I.: The polychromate and a chord diagram polynomial. Ann. Comb. 4, 227-236 (2000)

32. Schmitt, W.: Incidence Hopf algebras. J. Pure Appl. Algebra 96, 299-330 (1994)

33. Sidman, J.: On the Castelnuovo-Mumford regularity of subspace arrangements. Ph.D. Thesis, University of Michigan, 2002 
34. Speyer, D.E.: A Matroid invariant via the $K$-theory of the Grassmannian. Preprint (2006). arXiv:math.AG/0603551

35. Stanley, R.: A symmetric function generalization of the chromatic polynomial of a graph. Advances in Math. 111, 166-194 (1995)

36. Terao, H.: Generalized exponents of a free arrangement of hyperplanes and Shepard-Todd-Brieskorn formula. Invent. Math. 63, 159-179 (1981)

37. Tutte, W.T.: A contribution to the theory of chromatic polynomials. Canadian J. Math. 6, 80-91 (1954)

38. Welch, D.J.A.: Matroid Theory. Academic Press, New York (1976)

39. White, N.: Matroid Applications. Encyclopedia of Mathematics and its Applications, vol. 40. Cambridge University Press, Cambridge (1992)

40. Zaslavsky, T.: Counting the faces of cut-up spaces. Bull. Amer. Math. Soc. 81(5), 916-918 (1975) 